\title{
Almost Periodic Solutions for Neutral-Type BAM Neural Networks with Delays on Time Scales
}

\author{
Yongkun Li and Li Yang \\ Department of Mathematics, Yunnan University, Kunming, Yunnan 650091, China \\ Correspondence should be addressed to Yongkun Li; yklie@ynu.edu.cn
}

Received 5 May 2013; Accepted 17 June 2013

Academic Editor: Sabri Arik

Copyright ( 2013 Y. Li and L. Yang. This is an open access article distributed under the Creative Commons Attribution License, which permits unrestricted use, distribution, and reproduction in any medium, provided the original work is properly cited.

Using the existence of the exponential dichotomy of linear dynamic equations on time scales, a fixed point theorem and the theory of calculus on time scales, we obtain some sufficient conditions for the existence and exponential stability of almost periodic solutions for a class of neutral-type BAM neural networks with delays on time scales. Finally, a numerical example illustrates the feasibility of our results and also shows that the continuous-time neural network and its discrete-time analogue have the same dynamical behaviors. The results of this paper are completely new even if the time scale $\mathbb{T}=\mathbb{R}$ or $\mathbb{Z}$ and complementary to the previously known results.

\section{Introduction}

The bidirectional associative memory (BAM) neural network, which was introduced by Kosko (see [1]), is a special recurrent neural network that can store bipolar vector pairs and is composed of neurons arranged in two layers. The neurons in one layer are fully interconnected to the neurons in the other layer, while there are no interconnections among neurons in the same layer.

Recently, due to its wide range of applications, for instance, pattern recognition, associative memory, and combinatorial optimization, BAM neural network has received much attention. For example, in [2-4], some sufficient conditions were obtained for the stability of the equilibrium points of BAM neural networks; in $[5,6]$, authors investigated the periodic solutions of BAM neural networks by using the continuation theorem of coincidence degree theory; in [7-9], authors studied the almost periodic solution for BAM neural networks by using the exponential dichotomy and fixed point theorems; for other results about BAM neural networks, the reader may see [10-13] and reference therein.

Since it is natural and important that systems will contain some information about the derivative of the past state to further describe and model the dynamics for such complex neural reactions [14], many authors investigated the dynamical behaviors of neutral-type neural networks with delays
[15-26]. For example, in [21], under the assumptions that the activation functions satisfy boundedness and Lipschitz conditions, authors discussed global asymptotic stability of neutral-type BAM neural networks with delays as follows:

$$
\begin{array}{r}
x_{i}^{\prime}(t)+\sum_{j=1}^{m} e_{i j} x_{j}^{\prime}(t-h)=-a_{i} x_{i}(t)-\sum_{j=1}^{m} s_{i j} f_{j}\left(y_{j}(t-\tau)\right)+I_{i}, \\
i=1,2, \ldots, m, \\
y_{j}^{\prime}(t)+\sum_{i=1}^{m} v_{j i} y_{j}^{\prime}(t-d)=-c_{j} y_{j}(t)-\sum_{i=1}^{m} t_{j i} g_{i}\left(x_{i}(t-\delta)\right)+J_{j}, \\
j=1,2, \ldots, m .
\end{array}
$$

Also, it is well known that the study of dynamical systems on time scales is now an active area of research. One of the reasons for this is the fact that the study on time scales unifies the study of both discrete and continuous processes, besides many others. The pioneering works in this direction are [27-30]. The theory of time scales was initiated by Stefan Hilger in his Ph.D. thesis in 1988, providing a rich theory that unifies and extends discrete and continuous analysis [31, 32]. The time scales calculus has a tremendous potential for applications in some mathematical models of real processes 
and phenomena studied in physics, chemical technology, population dynamics, biotechnology and economics, neural networks, and social sciences.

In fact, both continuous and discrete systems are very important in implementation and applications. But, it is troublesome to study the the dynamical properties for continuous and discrete systems, respectively. Therefore, it is meaningful to study that on time scale which can unify the continuous and discrete situations (see [13,31,33-40]).

Motivated by the above, in this paper, we propose a neutral-type BAM neural network with delays on time scales as follows:

$$
\begin{gathered}
x_{i}^{\Delta}(t)=-a_{i}(t) x_{i}(t)+\sum_{j=1}^{m} a_{j i}(t) f_{j}\left(y_{j}\left(t-\tau_{j i}(t)\right)\right) \\
+\sum_{j=1}^{m} p_{j i}(t) g_{j}\left(y_{j}^{\Delta}\left(t-\sigma_{j i}(t)\right)\right)+I_{i}(t), \\
t \in \mathbb{T}, i=1,2, \ldots, n, \\
y_{j}^{\Delta}(t)=-b_{j}(t) y_{j}(t)+\sum_{i=1}^{n} b_{i j}(t) h_{i}\left(x_{i}\left(t-\zeta_{i j}(t)\right)\right) \\
+\sum_{i=1}^{n} q_{i j}(t) k_{i}\left(x_{i}^{\Delta}\left(t-\varsigma_{i j}(t)\right)\right)+J_{j}(t), \\
t \in \mathbb{T}, j=1,2, \ldots, m,
\end{gathered}
$$

where $\mathbb{T}$ is an almost periodic time scale; $n, m$ are the number of neurons in layers; $x_{i}(t)$ and $y_{j}(t)$ denote the activations of the $i$ th neuron and the $j$ th neuron at time $t ; a_{i}$ and $b_{j}$ represent the rate with which the $i$ th neuron and $j$ th neuron will reset their potential to the resting state in isolation when they are disconnected from the network and the external inputs at time $t ; f_{j}, g_{j}, h_{i}$, and $k_{i}$ are the input-output functions (the activation functions); $\tau_{j i}, \sigma_{j i}, \zeta_{i j}$, and $\varsigma_{i j}$ are transmission delays at time $t$ and satisfy $t-\tau_{j i}(t) \in \mathbb{T}, t-\sigma_{j i}(t) \in \mathbb{T}$, $t-\zeta_{i j}(t) \in \mathbb{T}$, and $t-\varsigma_{i j}(t) \in \mathbb{T}$ for $t \in \mathbb{T} ; a_{j i}, b_{i j}$ are elements of feedback templates at time $t ; p_{j i}, q_{i j}$ are elements of feed-forward templates at time $t$; and $I_{i}, J_{j}$ denote biases of the $i$ th neuron and the $j$ th neuron at time $t, i=1,2, \ldots, n$, $j=1,2, \ldots, m$.

Our main purpose of this paper is, using the exponential dichotomy of linear dynamic equations on time scales, a fixed point theorem and the theory of calculus on time scales, to study the existence and exponential stability of almost periodic solutions for (2). Our results of this paper are new and complementary to the previously known results even if the time scale $\mathbb{T}=\mathbb{R}$ or $\mathbb{Z}$.

For convenience, we denote $[a, b]_{\mathbb{T}}=\{t \mid t \in[a, b] \cap$ $\mathbb{T}\}$. For an almost periodic function $f: \mathbb{T} \rightarrow \mathbb{R}$, denote $f^{+}=\sup _{t \in \mathbb{T}}|f(t)|, f^{-}=\inf _{t \in \mathbb{T}}|f(t)|$. Set that $\mathbb{X}=\{\phi=$ $\left(\varphi_{1}, \varphi_{2}, \ldots, \varphi_{n}, \psi_{1}, \psi_{2}, \ldots, \psi_{m}\right)^{T} \mid \varphi_{i}, \psi_{j} \in C^{1}(\mathbb{T}, \mathbb{R}), \varphi_{i}, \psi_{j}$ are almost periodic functions on $\mathbb{T}, i=1,2, \ldots, n, j=$ $1,2, \ldots, m\}$ with the norm $\|\phi\|=\max \left\{|\varphi|_{1},|\psi|_{1}\right\}$, where $|\varphi|_{1}=\max \left\{|\varphi|_{0},\left|\varphi^{\Delta}\right|_{0}\right\},|\psi|_{1}=\max \left\{|\psi|_{0},\left|\psi^{\Delta}\right|_{0}\right\},|\varphi|_{0}=$ $\max _{1 \leq i \leq n} \varphi_{i}^{+},\left|\varphi^{\Delta}\right|_{0}=\max _{1 \leq i \leq n}\left(\varphi_{i}^{\Delta}(t)\right)^{+},|\psi|_{0}=\max _{1 \leq j \leq m} \psi_{j}^{+}$, and $\left|\psi^{\Delta}\right|_{0}=\max _{1 \leq j \leq m}\left(\psi_{j}^{\Delta}(t)\right)^{+}$, and $C^{1}(\mathbb{T}, \mathbb{R})$ is the set of continuous functions with continuous derivatives on $\mathbb{T}$, then $\mathbb{X}$ is a Banach space.

The initial condition of (2) is

$$
x_{i}(s)=\varphi_{i}(s), \quad y_{j}(s)=\psi_{j}(s), \quad s \in[-\theta, 0]_{\mathbb{T}},
$$

where $\theta=\max \left\{\max _{(i, j)}\left\{\tau_{j i}^{+}, \sigma_{j i}^{+}, \zeta_{i j}^{+}, \varsigma_{i j}^{+}\right\}\right\}, \varphi_{i}, \psi_{j} \in C^{1}\left([-\theta, 0]_{\mathbb{V}}\right.$, $\mathbb{R}), i=1,2, \ldots, n, j=1,2, \ldots, m$.

Throughout this paper, we assume that the following conditions hold:

$$
\begin{array}{r}
\left(\mathrm{H}_{1}\right) a_{j i}, p_{j i}, b_{i j}, q_{i j}, I_{i}, J_{j} \in C(\mathbb{T}, \mathbb{R}), a_{i}, b_{j} \in C\left(\mathbb{T}, \mathbb{R}^{+}\right) \text {with } \\
-a_{i},-b_{j} \in \mathscr{R}^{+}, \tau_{j i}, \sigma_{j i}, \zeta_{i j}, \text { and } \varsigma_{i j} \in C\left(\mathbb{T}, \mathbb{T} \cap \mathbb{R}^{+}\right) \text {are }
\end{array}
$$
all almost periodic functions, where $\mathscr{R}^{+}$denotes the set of positively regressive functions from $\mathbb{T}$ to $\mathbb{R}, i=$ $1,2, \ldots, n, j=1,2, \ldots, m$;

$\left(\mathrm{H}_{2}\right) f_{j}, g_{j}, h_{i}, k_{i} \in C(\mathbb{R}, \mathbb{R})$, and there exist positive constants $H_{j}^{f}, H_{j}^{g}, H_{i}^{h}$, and $H_{i}^{k}$ such that

$$
\begin{gathered}
\left|f_{j}(u)-f_{j}(v)\right| \leq H_{j}^{f}|u-v|, \\
\left|g_{j}(u)-g_{j}(v)\right| \leq H_{j}^{g}|u-v|, \\
\left|h_{i}(u)-h_{i}(v)\right| \leq H_{i}^{h}|u-v|, \\
\left|k_{i}(u)-k_{i}(v)\right| \leq H_{i}^{k}|u-v|,
\end{gathered}
$$

for all $u, v \in \mathbb{R}, i=1,2, \ldots, n, j=1,2, \ldots, m$.

This paper is organized as follows. In Section 2, we introduce some notations and definitions and state some preliminary results which are needed in later sections. In Section 3, we establish some sufficient conditions for the existence of almost periodic solutions of (2). In Section 4, we prove that the almost periodic solution obtained in Section 3 is exponentially stable. In Section 5, we give an example to illustrate the feasibility of our results obtained in previous sections. We draw a conclusion in Section 6.

\section{Preliminaries}

In this section, we introduce some definitions and state some preliminary results.

Definition 1 (see [32]). Let $\mathbb{T}$ be a nonempty closed subset (time scale) of $\mathbb{R}$. The forward and backward jump operators $\sigma, \rho: \mathbb{T} \rightarrow \mathbb{T}$ and the graininess $\mu: \mathbb{T} \rightarrow \mathbb{R}_{+}$are defined, respectively, by

$$
\begin{gathered}
\sigma(t)=\inf \{s \in \mathbb{T}: s>t\}, \\
\rho(t)=\sup \{s \in \mathbb{T}: s<t\}, \\
\mu(t)=\sigma(t)-t .
\end{gathered}
$$

Lemma 2 (see [32]). Assume that $p, q: \mathbb{T} \rightarrow \mathbb{R}$ are two regressive functions, then 
(i) $e_{0}(t, s) \equiv 1$ and $e_{p}(t, t) \equiv 1$;

(ii) $e_{p}(t, s)=1 / e_{p}(s, t)=e_{\ominus p}(s, t)$;

(iii) $e_{p}(t, s) e_{p}(s, r)=e_{p}(t, r)$;

(iv) $\left(e_{p}(t, s)\right)^{\Delta}=p(t) e_{p}(t, s)$.

Lemma 3 (see [32]). Let $f, g$ be $\Delta$-differentiable functions on T. Then

(i) $\left(\nu_{1} f+v_{2} g\right)^{\Delta}=v_{1} f^{\Delta}+v_{2} g^{\Delta}$, for any constants $\nu_{1}, v_{2}$;

(ii) $(f g)^{\Delta}(t)=f^{\Delta}(t) g(t)+f(\sigma(t)) g^{\Delta}(t)=f(t) g^{\Delta}(t)+$ $f^{\Delta}(t) g(\sigma(t))$.

Lemma 4 (see [32]). Assume that $p(t) \geq 0$ for $t \geq s$. Then $e_{p}(t, s) \geq 1$.

Definition 5 (see [32]). A function $f: \mathbb{T} \rightarrow \mathbb{R}$ is positively regressive if $1+\mu(t) f(t)>0$ for all $t \in \mathbb{T}$.

Lemma 6 (see [32]). Suppose that $p \in \mathscr{R}^{+}$. Then

(i) $e_{p}(t, s)>0$, for all $t, s \in \mathbb{T}$;

(ii) if $p(t) \leq q(t)$ for all $t \geq s, t, s \in \mathbb{T}$, then $e_{p}(t, s) \leq$ $e_{q}(t, s)$ for all $t \geq s$.

Lemma 7 (see [32]). If $p \in \mathscr{R}$ and $a, b, c \in \mathbb{T}$, then

$$
\begin{gathered}
{\left[e_{p}(c, \cdot)\right]^{\Delta}=-p\left[e_{p}(c, \cdot)\right]^{\sigma},} \\
\int_{a}^{b} p(t) e_{p}(c, \sigma(t)) \Delta t=e_{p}(c, a)-e_{p}(c, b) .
\end{gathered}
$$

Lemma 8 (see [32]). Let $a \in \mathbb{T}^{k}, b \in \mathbb{T}$, and assume that $f: \mathbb{T} \times \mathbb{T}^{k} \rightarrow \mathbb{R}$ is continuous at $(t, t)$, where $t \in \mathbb{T}^{k}$ with $t>a$. Also assume that $f^{\Delta}(t, \cdot)$ is $r d$-continuous on $[a, \sigma(t)]$. Suppose that for each $\varepsilon>0$, there exists a neighborhood $U$ of $\tau \in[a, \sigma(t)]$ such that

$$
\begin{array}{r}
\left|f(\sigma(t), \tau)-f(s, \tau)-f^{\Delta}(t, \tau)(\sigma(t)-s)\right| \leq \varepsilon|\sigma(t)-s|, \\
\forall s \in U,
\end{array}
$$

where $f^{\Delta}$ denotes the derivative of $f$ with respect to the first variable. Then,

(i) $g(t):=\int_{a}^{t} f(t, \tau) \Delta \tau$ implies $g^{\Delta}(t):=\int_{a}^{t} f^{\Delta}(t, \tau) \Delta \tau+$ $f(\sigma(t), t)$;

(ii) $h(t):=\int_{t}^{b} f(t, \tau) \Delta \tau$ implies $h^{\Delta}(t):=\int_{t}^{b} f^{\Delta}(t, \tau) \Delta \tau-$ $f(\sigma(t), t)$.

Definition 9 (see [34]). A time scale $\mathbb{T}$ is called an almost periodic time scale if

$$
\Pi:=\{\tau \in \mathbb{R}: t+\tau \in \mathbb{T}, \forall t \in \mathbb{T}\} \neq\{0\} .
$$

Definition 10 (see [34]). Let $\mathbb{T}$ be an almost periodic time scale. A function $f(t): \mathbb{T} \rightarrow \mathbb{R}^{n}$ is said to be almost periodic on $\mathbb{T}$, if for any $\varepsilon>0$, the set

$$
E(\varepsilon, f)=\{\tau \in \Pi:|f(t+\tau)-f(t)|<\varepsilon, \forall t \in \mathbb{T}\}
$$

is relatively dense in $\mathbb{T}$; that is, for any $\varepsilon>0$, there exists a constant $l(\varepsilon)>0$ such that each interval of length $l(\varepsilon)$ contains at least one $\tau \in E(\varepsilon, f)$ such that

$$
|f(t+\tau)-f(t)|<\varepsilon, \quad \forall t \in \mathbb{T} .
$$

The set $E(\varepsilon, f)$ is called the $\varepsilon$-translation set of $f(t), \tau$ is called the $\varepsilon$-translation number of $f(t)$, and $l(\varepsilon)$ is called the inclusion of $E(\varepsilon, f)$.

Lemma 11 (see [34]). If $f \in C\left(\mathbb{T}, \mathbb{R}^{n}\right)$ is an almost periodic function, then $f$ is bounded on $\mathbb{T}$.

Lemma 12 (see [34]). If $f, g \in\left(\mathbb{T}, \mathbb{R}^{n}\right)$ are almost periodic functions, then $f+g, f g$ are also almost periodic.

Definition 13 (see [35]). Letting $X \in \mathbb{R}^{n}$ and $A(t)$ be a $n \times n$ matrix-valued function on $\mathbb{T}$, the linear system

$$
X^{\Delta}(t)=A(t) X(t), \quad t \in \mathbb{T}
$$

is said to admit an exponential dichotomy on $\mathbb{T}$ if there exist positive constants $k_{i}, \alpha_{i}, i=1,2$, projection $P$, and the fundamental solution matrix $X(t)$ of (11) satisfying

$$
\begin{gathered}
\left|X(t) P X^{-1}(s)\right| \leq k_{1} e_{\ominus \alpha_{1}}(t, s), \quad s, t \in \mathbb{T}, t \geq s, \\
\left|X(t)(I-P) X^{-1}(s)\right| \leq k_{2} e_{\ominus \alpha_{2}}(s, t), \quad s, t \in \mathbb{T}, t \leq s,
\end{gathered}
$$

where $|\cdot|$ is a matrix norm on $\mathbb{T}$; that is, if $A=\left(a_{i j}\right)_{n \times m}$, then we can take $|A|=\left(\sum_{i=1}^{n} \sum_{j=1}^{m}\left|a_{i j}\right|^{2}\right)^{1 / 2}$.

Lemma 14 (see [34]). If (11) admits an exponential dichotomy, then the following almost periodic system

$$
X^{\Delta}(t)=A(t) X(t)+g(t), \quad t \in \mathbb{T}
$$

has an almost periodic solution as follows:

$$
\begin{aligned}
X(t)= & \int_{-\infty}^{t} X(t) P X^{-1}(\sigma(s)) g(s) \Delta s \\
& -\int_{t}^{+\infty} X(t)(I-P) X^{-1}(\sigma(s)) g(s) \Delta s,
\end{aligned}
$$

where $X(t)$ is the fundamental solution matrix of (11).

Lemma 15 (see [35]). If $A(t)$ is a uniformly bounded $r d$ continuous $n \times n$ matrix-valued function on $\mathbb{T}$, and there is a $\delta>0$ such that

$$
\begin{array}{r}
\left|a_{i i}(t)\right|-\sum_{j \neq i}\left|a_{i j}(t)\right|-\frac{1}{2} \mu(t)\left(\sum_{j=1}^{n}\left|a_{i j}(t)\right|\right)^{2}-\delta^{2} \mu(t) \geq 2 \delta, \\
t \in \mathbb{T}, i=1,2, \ldots, n,
\end{array}
$$

then (11) admits an exponential dichotomy on $\mathbb{T}$. 
Definition 16. Let $z^{*}(t)=\left(x_{1}^{*}(t), x_{2}^{*}(t), \ldots, x_{n}^{*}(t), y_{1}^{*}(t)\right.$, $\left.y_{2}^{*}(t), \ldots, y_{m}^{*}(t)\right)^{T}$ be an almost periodic solution of (2) with initial value $\phi^{*}(s)=\left(\varphi_{1}^{*}(s), \varphi_{2}^{*}(s), \ldots, \varphi_{n}^{*}(s), \psi_{1}^{*}(s), \psi_{2}^{*}(s), \ldots\right.$, $\left.\psi_{m}^{*}(s)\right)^{T}$. If there exists a positive constant $\lambda$ with $-\lambda \epsilon$ $\mathscr{R}^{+}$such that for $t_{0} \in[-\theta, 0]_{\mathbb{T}}$, there exists $M>1$ such that for an arbitrary solution $z(t)=\left(x_{1}(t), x_{2}(t), \ldots, x_{n}(t), y_{1}(t)\right.$, $\left.y_{2}(t), \ldots, y_{m}(t)\right)^{T}$ of (2) with initial value $\phi(s)=\left(\varphi_{1}(s)\right.$, $\left.\varphi_{2}(s), \ldots, \varphi_{n}(s), \psi_{1}(s), \psi_{2}(s), \ldots, \psi_{m}(s)\right)^{T}$ satisfies

$$
\begin{array}{r}
\left|z(t)-z^{*}(t)\right|_{1} \leq M\left\|\phi-\phi^{*}\right\| e_{-\lambda}\left(t, t_{0}\right), \\
t \in[-\theta, \infty)_{\mathbb{T}}, t \geq t_{0},
\end{array}
$$

where $\left|z(t)-z^{*}(t)\right|_{1}=\max \left\{\max _{1 \leq i \leq n}\left\{\left|x_{i}(t)-x_{i}^{*}(t)\right|, \mid\left(x_{i}(t)-\right.\right.\right.$ $\left.\left.\left.x_{i}^{*}(t)\right)^{\Delta} \mid\right\}, \max _{1 \leq j \leq m}\left\{\left|y_{j}(t)-y_{j}^{*}(t)\right|,\left|\left(y_{j}(t)-y_{j}^{*}(t)\right)^{\Delta}\right|\right\}\right\}, \| \phi-$

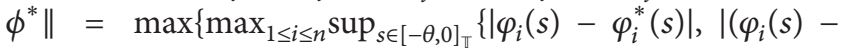
$\left.\left.\varphi_{i}^{*}(s)\right)^{\Delta} \mid\right\}, \max _{1 \leq j \leq m} \sup _{s \in[-\theta, 0]_{T}}\left\{\left|\psi_{j}(s)-\psi_{j}^{*}(s)\right|, \mid\left(\psi_{j}(s)-\right.\right.$ $\left.\left.\left.\psi_{j}^{*}(s)\right)^{\Delta} \mid\right\}\right\}$. Then, the solution $z^{*}(t)$ is said to be exponentially stable.

\section{Existence of Almost Periodic Solutions}

In this section, we will state and prove the sufficient conditions for the existence of almost periodic solutions of (2).

Let $\phi^{0}(t)=\left(\varphi_{1}^{0}(t), \varphi_{2}^{0}(t), \ldots, \varphi_{n}^{0}(t), \psi_{1}^{0}(t), \psi_{2}^{0}(t), \ldots\right.$, $\left.\psi_{m}^{0}(t)\right)^{T}$, where $\varphi_{i}^{0}(t)=\int_{-\infty}^{t} e_{-a_{i}}(t, \sigma(s)) \times I_{i}(s) \Delta s, \psi_{j}^{0}(t)=$ $\int_{-\infty}^{t} e_{-b_{j}}(t, \sigma(s)) J_{j}(s) \Delta s, i=1,2, \ldots, n, j=1,2, \ldots, m$, and let $L$ be a constant satisfying $L \geq \max \left\{\left\|\phi^{0}\right\|\right.$, $\max _{1 \leq j \leq m}\left\{\left|f_{j}(0)\right|,\left|g_{j}(0)\right|, \max _{1 \leq i \leq n}\left\{\left|h_{i}(0)\right|, k_{i}(0) \mid\right\}\right.$. We have the following theorem.

Theorem 17. Let $\left(H_{1}\right)$ and $\left(H_{2}\right)$ hold. Suppose that

$\left(\mathrm{H}_{3}\right)$ there exists a positive constant $\delta$ such that

$$
\begin{array}{r}
c_{k}(t)-\frac{1}{2} \mu(t) c_{k}^{2}(t)-\delta^{2} \mu(t) \geq 2 \delta, \\
t \in \mathbb{T}, k=1,2, \ldots, n+m,
\end{array}
$$

where

$$
c_{k}(t)= \begin{cases}a_{i}(t), & k=i, i=1,2, \ldots, n, \\ b_{j}(t), & k=n+j, \quad j=1,2, \ldots, m ;\end{cases}
$$

$\left(\mathrm{H}_{4}\right) \max \left\{\max _{1 \leq i \leq n}\left\{\left(\theta_{i} / a_{i}^{-}\right), \quad\left(1+\left(a_{i}^{+} / a_{i}^{-}\right)\right) \theta_{i}\right\}\right.$, $\left.\max _{1 \leq j \leq m}\left\{\left(\gamma_{j} / b_{j}^{-}\right),\left(1+\left(b_{j}^{+} / b_{j}^{-}\right)\right) \gamma_{j}\right\}\right\} \leq(1 / 2)$, where $\theta_{i}=\sum_{j=1}^{m}\left(a_{j i}^{+} H_{j}^{f}+p_{j i}^{+} H_{j}^{g}+a_{j i}^{+}+p_{j i}^{+}\right)$, $\gamma_{j}=\sum_{i=1}^{n}\left(b_{i j}^{+} H_{i}^{h}+q_{i j}^{+} H_{i}^{k}+b_{i j}^{+}+q_{i j}^{+}\right), i=1,2$, $\ldots, n, j=1,2, \ldots, m$.

Then, (2) has a unique almost periodic solution in $\mathbb{X}_{0}=$ $\left\{\phi \in \mathbb{X} \mid\left\|\phi-\phi^{0}\right\| \leq L\right\}, \phi(t)=\left(\varphi_{1}(t), \varphi_{2}(t), \ldots, \varphi_{n}(t)\right.$, $\left.\psi_{1}(t), \psi_{2}(t), \ldots, \psi_{m}(t)\right)^{T}$.
Proof. For any given $\phi \in \mathbb{X}$, we consider the following almost periodic system:

$$
\begin{array}{cc}
x_{i}^{\Delta}(t)=-a_{i}(t) x_{i}(t)+F_{i}(t, \psi)+I_{i}(t), & i=1,2, \ldots, n, \\
y_{j}^{\Delta}(t)=-b_{j}(t) y_{j}(t)+G_{j}(t, \varphi)+J_{j}(t), & j=1,2, \ldots, m,
\end{array}
$$

where

$$
\begin{aligned}
F_{i}(t, \psi)= & \sum_{j=1}^{m} a_{j i}(t) f_{j}\left(\psi_{j}\left(t-\tau_{j i}(t)\right)\right) \\
& +\sum_{j=1}^{m} p_{j i}(t) g_{j}\left(\psi_{j}^{\Delta}\left(t-\sigma_{j i}(t)\right)\right), \quad i=1,2, \ldots, n, \\
G_{j}(t, \varphi)= & \sum_{i=1}^{n} b_{i j}(t) h_{i}\left(\varphi_{i}\left(t-\zeta_{i j}(t)\right)\right) \\
& +\sum_{i=1}^{n} q_{i j}(t) k_{i}\left(\varphi_{i}^{\Delta}\left(t-\zeta_{i j}(t)\right)\right), \quad j=1,2, \ldots, m .
\end{aligned}
$$

Since $\left(\mathrm{H}_{3}\right)$ holds, it follows from Lemma 15 that the linear system

$$
\begin{array}{ll}
x_{i}^{\Delta}(t)=-a_{i}(t) x_{i}(t), & i=1,2, \ldots, n, \\
y_{j}^{\Delta}(t)=-b_{j}(t) y_{j}(t), & j=1,2, \ldots, m
\end{array}
$$

admits an exponential dichotomy on $\mathbb{T}$. Thus, by Lemma 14, we obtain that (19) has an almost periodic solution, which is expressed as follows:

$$
\begin{array}{r}
x_{i}^{\varphi}(t)=\int_{-\infty}^{t} e_{-a_{i}}(t, \sigma(s))\left(F_{i}(s, \psi)+I_{i}(s)\right) \Delta s, \\
i=1,2, \ldots, n, \\
y_{j}^{\psi}(t)=\int_{-\infty}^{t} e_{-b_{j}}(t, \sigma(s))\left(G_{j}(s, \varphi)+J_{i}(s)\right) \Delta s, \\
j=1,2, \ldots, m .
\end{array}
$$

For $\phi \in \mathbb{X}_{0}$, then $\|\phi\| \leq\left\|\phi-\phi_{0}\right\|+\left\|\phi_{0}\right\| \leq 2 L$. Define the following operator:

$$
\begin{gathered}
\Phi: \mathbb{X}_{0} \longrightarrow \mathbb{X}_{0} \\
\left(\varphi_{1}, \varphi_{2}, \ldots, \varphi_{n}, \psi_{1}, \psi_{2}, \ldots, \psi_{m}\right)^{T} \\
\longrightarrow\left(x_{1}^{\varphi}, x_{2}^{\varphi}, \ldots, x_{n}^{\varphi}, y_{1}^{\psi}, y_{2}^{\psi}, \ldots, y_{m}^{\psi}\right)^{T}
\end{gathered}
$$

We will show that $\Phi$ is a contraction. 
First, we show that for any $\phi \in \mathbb{X}_{0}$, we have $\Phi \phi \in \mathbb{X}_{0}$. Note that

$$
\begin{aligned}
&\left|F_{i}(s, \psi)\right|= \mid \sum_{j=1}^{m} a_{j i}(s) f_{j}\left(\psi_{j}\left(s-\tau_{j i}(s)\right)\right) \\
&+\sum_{j=1}^{m} p_{j i}(s) g_{j}\left(\psi_{j}^{\Delta}\left(s-\sigma_{j i}(s)\right)\right) \mid \\
& \leq \sum_{j=1}^{m} a_{j i}^{+}\left(\left|f_{j}\left(\psi_{j}\left(s-\tau_{j i}(s)\right)\right)-f_{j}(0)\right|+\left|f_{j}(0)\right|\right) \\
&+\sum_{j=1}^{m} p_{j i}^{+}\left(\left|g_{j}\left(\psi_{j}^{\Delta}\left(s-\sigma_{j i}(s)\right)\right)-g_{j}(0)\right|\right. \\
& \leq \sum_{j=1}^{m}\left(a_{j i}^{+} H_{j}^{f}+p_{j i}^{+} H_{j}^{g}\right)|\psi|_{1} \\
&+\sum_{j=1}^{m}\left(a_{j i}^{+}\left|f_{j}(0)\right|+p_{j i}^{+}\left|g_{j}(0)\right|\right) \\
& \leq \sum_{j=1}^{m}\left(a_{j i}^{+} H_{j}^{f}+p_{j i}^{+} H_{j}^{g}\right)\|\phi\| \\
&+\sum_{j=1}^{m}\left(a_{j i}^{+}\left|f_{j}(0)\right|+p_{j i}^{+}\left|g_{j}(0)\right|\right) \\
& \leq \sum_{j=1}^{m}\left(a_{j i}^{+} H_{j}^{f}+p_{j i}^{+} H_{j}^{g}+a_{j i}^{+}+p_{j i}^{+}\right) 2 L:=2 \theta_{i} L, \\
& i=1,2, \ldots, n
\end{aligned}
$$

and similarly,

$$
\begin{array}{r}
\left|G_{j}(s, \varphi)\right| \leq \sum_{i=1}^{n}\left(b_{i j}^{+} H_{i}^{h}+q_{i j}^{+} H_{i}^{k}+b_{i j}^{+}+q_{i j}^{+}\right) 2 L:=2 \gamma_{j} L, \\
j=1,2, \ldots, m .
\end{array}
$$

Therefore, we have

$$
\begin{aligned}
\left|\left(\Phi\left(\phi-\phi^{0}\right)\right)_{i}(t)\right| & =\left|\int_{-\infty}^{t} e_{-a_{i}}(t, \sigma(s)) F_{i}(s, \psi) \Delta s\right| \\
& \leq \int_{-\infty}^{t} e_{-a_{i}}(t, \sigma(s))\left|F_{i}(s, \psi)\right| \Delta s \\
& \leq \int_{-\infty}^{t} e_{-a_{i}^{-}}(t, \sigma(s)) 2 \theta_{i} L \Delta s \\
& \leq \frac{2 \theta_{i} L}{a_{i}^{-}}, \quad i=1,2, \ldots, n,
\end{aligned}
$$

$$
\begin{aligned}
\left|\left(\Phi\left(\phi-\phi^{0}\right)\right)_{n+j}(t)\right| & =\left|\int_{-\infty}^{t} e_{-b_{j}}(t, \sigma(s)) G_{j}(s, \varphi) \Delta s\right| \\
& \leq \int_{-\infty}^{t} e_{-b_{j}}(t, \sigma(s))\left|G_{j}(s, \varphi)\right| \Delta s \\
& \leq \int_{-\infty}^{t} e_{-b_{j}^{-}}(t, \sigma(s)) 2 \gamma_{j} L \Delta s \\
& \leq \frac{2 \gamma_{j} L}{b_{j}^{-}}, \quad j=1,2, \ldots, m .
\end{aligned}
$$

On the other hand, for $i=1,2, \ldots, n$, we have

$$
\begin{aligned}
& \left|\left(\Phi\left(\phi-\phi^{0}\right)\right)_{i}^{\Delta}(t)\right| \\
= & \left|\left(\int_{-\infty}^{t} e_{-a_{i}}(t, \sigma(s)) F_{i}(s, \psi) \Delta s\right)_{t}^{\Delta}\right| \\
= & \left|F_{i}(t, \psi)-a_{i}(t) \int_{-\infty}^{t} e_{-a_{i}}(t, \sigma(s)) F_{i}(s, \psi) \Delta s\right| \\
\leq & \left|F_{i}(t, \psi)\right|+\left|a_{i}(t)\right| \\
& \times \int_{-\infty}^{t} e_{-a_{i}}(t, \sigma(s))\left|F_{i}(s, \psi)\right| \Delta s \\
\leq & \left(1+\frac{a_{i}^{+}}{a_{i}^{-}}\right) 2 \theta_{i} L
\end{aligned}
$$

and for $j=1,2, \ldots, m$,

$$
\left|\left(\Phi\left(\phi-\phi^{0}\right)\right)_{n+j}^{\Delta}(t)\right| \leq\left(1+\frac{b_{j}^{+}}{b_{j}^{-}}\right) 2 \gamma_{j} L .
$$

In view of $\left(\mathrm{H}_{4}\right)$, we have

$$
\begin{aligned}
&\left\|\Phi \phi-\phi^{0}\right\| \leq \max \left\{\max _{1 \leq i \leq n}\left\{\frac{2 \theta_{i} L}{a_{i}^{-}},\left(1+\frac{a_{i}^{+}}{a_{i}^{-}}\right) 2 \theta_{i} L\right\},\right. \\
&\left.\max _{1 \leq j \leq m}\left\{\frac{2 \gamma_{j} L}{b_{j}^{-}},\left(1+\frac{b_{j}^{+}}{b_{j}^{-}}\right) 2 \gamma_{j} L\right\}\right\} \leq L,
\end{aligned}
$$

that is, $\Phi \phi \in \mathbb{X}_{0}$. Next, we show that $\Phi$ is a contraction. For $\phi=\left(\varphi_{1}, \varphi_{2}, \ldots, \varphi_{n}, \psi_{1}, \psi_{2}, \ldots, \psi_{m}\right)^{T}, \vartheta=$ $\left(\xi_{1}, \xi_{2}, \ldots, \xi_{n}, \eta_{1}, \eta_{2}, \ldots, \eta_{m}\right)^{T} \in \mathbb{X}_{0}$, for $i=1,2, \ldots, n$, denote by

$$
\begin{aligned}
& F_{i}^{(1)}(s, \psi, \eta) \\
& \quad=\sum_{j=1}^{m} a_{j i}(s)\left(f_{j}\left(\psi_{j}\left(s-\tau_{j i}(s)\right)\right)-f_{j}\left(\eta_{j}\left(s-\tau_{j i}(s)\right)\right)\right), \\
& F_{i}^{(2)}(s, \psi, \eta) \\
& \quad=\sum_{j=1}^{m} p_{j i}(s)\left(g_{j}\left(\psi_{j}^{\Delta}\left(s-\sigma_{j i}(s)\right)\right)-g_{j}\left(\eta_{j}^{\Delta}\left(s-\sigma_{j i}(s)\right)\right)\right) .
\end{aligned}
$$


Then, we have

$$
\begin{aligned}
& \left|(\Phi \phi-\Phi \zeta)_{i}(t)\right| \\
& =\left|\int_{-\infty}^{t} e_{-a_{i}}(t, \sigma(s))\left(F_{i}^{(1)}(s, \psi, \eta)+F_{i}^{(2)}(s, \psi, \eta)\right) \Delta s\right| \\
& \leq \int_{-\infty}^{t} e_{-a_{i}}(t, \sigma(s)) \\
& \times\left(\sum_{j=1}^{m} a_{j i}^{+} H_{j}^{f}\left|\psi_{j}\left(s-\tau_{j i}(s)\right)-\eta_{j}\left(s-\tau_{j i}(s)\right)\right|\right. \\
& +\sum_{j=1}^{m} p_{j i}^{+} H_{j}^{g} \mid \psi_{j}^{\Delta}\left(s-\sigma_{j i}(s)\right) \\
& \left.-\eta_{j}^{\Delta}\left(s-\sigma_{j i}(s)\right) \mid\right) \Delta s
\end{aligned}
$$$$
\leq \frac{1}{a_{i}^{-}} \sum_{j=1}^{m}\left(a_{j i}^{+} H_{j}^{f}+p_{j i}^{+} H_{j}^{g}\right)|\psi-\eta|_{1}, \quad i=1,2, \ldots, n,
$$$$
\left|(\Phi \phi-\Phi \zeta)_{i}^{\Delta}(t)\right|
$$$$
=\left|\left(\int_{-\infty}^{t} e_{-a_{i}}(t, \sigma(s))\left(F_{i}^{(1)}(s, \psi, \eta)+F_{i}^{(2)}(s, \psi, \eta)\right) \Delta s\right)_{t}^{\Delta}\right|
$$$$
=\mid F_{i}^{(1)}(t, \psi, \eta)+F_{i}^{(2)}(t, \psi, \eta)-a_{i}(t)
$$$$
\times \int_{-\infty}^{t} e_{-a_{i}}(t, \sigma(s))\left(F_{i}^{(1)}(s, \psi, \eta)+F_{i}^{(2)}(s, \psi, \eta)\right) \Delta s \mid
$$$$
\leq\left|F_{i}^{(1)}(t, \psi, \eta)\right|+\left|F_{i}^{(2)}(t, \psi, \eta)\right|+\left|a_{i}(t)\right|
$$$$
\times \int_{-\infty}^{t} e_{-a_{i}}(t, \sigma(s))\left|F_{i}^{(1)}(s, \psi, \eta)+F_{i}^{(2)}(s, \psi, \eta)\right| \Delta s
$$$$
\leq \sum_{j=1}^{m}\left(a_{j i}^{+} H_{j}^{f}+p_{j i}^{+} H_{j}^{g}\right)|\psi-\eta|_{1}
$$$$
+\frac{a_{i}^{+}}{a_{i}^{-}} \sum_{j=1}^{m}\left(a_{j i}^{+} H_{j}^{f}+p_{j i}^{+} H_{j}^{g}\right)|\psi-\eta|_{1}
$$$$
=\left(1+\frac{a_{i}^{+}}{a_{i}^{-}}\right) \sum_{j=1}^{m}\left(a_{j i}^{+} H_{j}^{f}+p_{j i}^{+} H_{j}^{g}\right)|\psi-\eta|_{1} \text {, }
$$$$
i=1,2, \ldots, n \text {. }
$$

In a similar way, we have

$$
\begin{aligned}
&\left|(\Phi \phi-\Phi \zeta)_{n+j}(t)\right| \leq \frac{1}{b_{j}^{-}} \sum_{i=1}^{n}\left(b_{i j}^{+} H_{i}^{h}+q_{i j}^{+} H_{i}^{k}\right)|\varphi-\xi|_{1}, \\
&\left|(\Phi \phi-\Phi \zeta)_{n+j}^{\Delta}(t)\right| \leq\left(1+\frac{b_{j}^{+}}{b_{j}^{-}}\right) \\
& \times \sum_{i=1}^{n}\left(b_{i j}^{+} H_{i}^{h}+q_{i j}^{+} H_{i}^{k}\right)|\varphi-\xi|_{1}, \\
& j=1,2, \ldots, m .
\end{aligned}
$$

By $\left(\mathrm{H}_{4}\right)$, we have

$$
\begin{aligned}
\max \left\{\max _{1 \leq i \leq n}\left\{\frac{1}{a_{i}^{-}} \varrho_{i},\left(1+\frac{a_{i}^{+}}{a_{i}^{-}}\right) \varrho_{i}\right\},\right. \\
\left.\max _{1 \leq j \leq m}\left\{\frac{1}{b_{j}^{-}} \rho_{j},\left(1+\frac{b_{j}^{+}}{b_{j}^{-}}\right) \rho_{j}\right\}\right\}<1,
\end{aligned}
$$

where $\varrho_{i}=\sum_{j=1}^{m}\left(a_{j i}^{+} H_{j}^{f}+p_{j i}^{+} H_{j}^{g}\right)$ and $\rho_{j}=\sum_{i=1}^{n}\left(b_{i j}^{+} H_{i}^{h}+\right.$ $\left.q_{i j}^{+} H_{i}^{k}\right), i=1,2, \ldots, n, j=1,2, \ldots, m$. It implies that $\| \Phi \phi-$ $\Phi \zeta\|<\| \varphi-\psi \|$. Hence, $\Phi$ is a contraction. Therefore, $\Phi$ has a fixed point in $\mathbb{X}_{0}$; that is, (2) has a unique almost periodic solution in $\mathbb{X}_{0}$. This completes the proof of Theorem 17 .

\section{Exponential Stability of Almost Periodic Solution}

In this section, we will study the exponential stability of almost periodic solution of (2).

Theorem 18. Let $\left(H_{1}\right)-\left(H_{4}\right)$ hold. Suppose further that

$$
\begin{gathered}
\left(\mathrm{H}_{5}\right) \varrho_{i} \geq\left(a_{i}^{-}-a_{i}^{+} a_{i}^{-}\right) /\left(a_{i}^{+}+a_{i}^{-}\right) \text {and } \rho_{j} \geq\left(b_{j}^{-}-b_{j}^{+} b_{j}^{-}\right) /\left(b_{j}^{+}+\right. \\
\left.b_{j}^{-}\right), \text {where } \varrho_{i}=\sum_{j=1}^{m}\left(a_{j i}^{+} H_{j}^{f}+p_{j i}^{+} H_{j}^{g}\right) \text { and } \rho_{j}= \\
\sum_{i=1}^{n}\left(b_{i j}^{+} H_{i}^{h}+q_{i j}^{+} H_{i}^{k}\right), i=1,2, \ldots, n, j=1,2, \ldots, m .
\end{gathered}
$$

Then, the almost periodic solution of (2) is exponentially stable.

Proof. By Theorem 17, (2) has an almost periodic solution $\omega(t)=\left(\alpha_{1}(t), \alpha_{2}(t), \ldots, \alpha_{n}(t), \beta_{1}(t), \beta_{2}(t), \ldots, \beta_{m}(t)\right)^{T}$ with initial condition $\phi^{*}(s)=\left(\varphi_{1}^{*}(s), \varphi_{2}^{*}(s), \ldots, \varphi_{n}^{*}(s), \psi_{1}^{*}(s)\right.$, $\left.\psi_{2}^{*}(s), \ldots, \psi_{m}^{*}(s)\right)^{T}$. Suppose that $z(t)=\left(x_{1}(t), x_{2}(t), \ldots\right.$, $\left.x_{n}(t), y_{1}(t), y_{2}(t), \ldots, y_{m}(t)\right)^{T}$ is an arbitrary solution of (2) with initial condition $\phi(s)=\left(\varphi_{1}(s), \varphi_{2}(s), \ldots, \varphi_{n}(s), \psi_{1}(s)\right.$, $\left.\psi_{2}(s), \ldots, \psi_{m}(s)\right)^{T}$. Denote $v(t)=\left(u_{1}(t), u_{2}(t), \ldots, u_{n}(t)\right.$, $\left.v_{1}(t), v_{2}(t), \ldots, v_{m}(t)\right)^{T}$, where $u_{i}(t)=x_{i}(t)-\alpha_{i}(t)$, 
$v_{j}(t)=y_{j}(t)-\beta_{j}(t), i=1,2, \ldots, n, j=1,2, \ldots, m$. Then, it follows from (2) that

$$
\begin{aligned}
& u_{i}^{\Delta}(t)=-a_{i}(t) u_{i}(t) \\
& +\sum_{j=1}^{m} a_{j i}(t)\left(f_{j}\left(y_{j}\left(t-\tau_{j i}(t)\right)\right)\right. \\
& \left.-f_{j}\left(\beta_{j}\left(t-\tau_{j i}(t)\right)\right)\right) \\
& +\sum_{j=1}^{m} p_{j i}(t)\left(g_{j}\left(y_{j}^{\Delta}\left(t-\sigma_{j i}(t)\right)\right)\right. \\
& \left.-g_{j}\left(\beta_{j}^{\Delta}\left(t-\sigma_{j i}(t)\right)\right)\right) \text {, } \\
& i=1,2, \ldots, n, \\
& v_{j}^{\Delta}(t)=-b_{j}(t) v_{j}(t) \\
& +\sum_{i=1}^{n} b_{i j}(t)\left(h_{i}\left(x_{i}\left(t-\zeta_{i j}(t)\right)\right)\right. \\
& \left.-h_{i}\left(\alpha_{i}\left(t-\zeta_{i j}(t)\right)\right)\right) \\
& +\sum_{i=1}^{n} q_{i j}(t)\left(k_{i}\left(x_{i}^{\Delta}\left(t-\varsigma_{i j}(t)\right)\right)\right. \\
& \left.-k_{i}\left(\alpha_{i}^{\Delta}\left(t-\varsigma_{i j}(t)\right)\right)\right), \\
& j=1,2, \ldots, m \text {. }
\end{aligned}
$$

The initial condition of (34) and (35) is

$$
\begin{gathered}
u_{i}(s)=\varphi_{i}(s)-\varphi_{i}^{*}(s), \\
v_{j}(s)=\psi_{j}(s)-\psi_{j}^{*}(s), \quad s \in[-\theta, 0]_{\mathbb{T}},
\end{gathered}
$$

where $i=1,2, \ldots, n, j=1,2, \ldots, m$.

Multiplying both sides of (34) by $e_{-a_{i}}(t, \sigma(s))$ and integrating on $\left[t_{0}, t\right]_{\mathbb{T}}$, where $t_{0} \in[-\theta, 0]_{\mathbb{T}}$, we get

$$
\begin{gathered}
u_{i}(t) \\
=u_{i}\left(t_{0}\right) e_{-a_{i}}\left(t, t_{0}\right) \\
+\int_{t_{0}}^{t} e_{-a_{i}}(t, \sigma(s)) \\
\quad \times\left\{\sum _ { j = 1 } ^ { m } a _ { j i } ( s ) \left(f_{j}\left(y_{j}\left(s-\tau_{j i}(s)\right)\right)\right.\right. \\
+\sum_{j=1}^{m} p_{j i}(s)\left(g_{j}\left(y_{j}^{\Delta}\left(s-\sigma_{j i}(s)\right)\right)\right. \\
\left.\left.-g_{j}\left(\beta_{j}^{\Delta}\left(s-\sigma_{j i}(s)\right)\right)\right)\right\} \Delta s, \\
i=1,2, \ldots, n .
\end{gathered}
$$

Similarly, multiplying both sides of (35) by $e_{-b_{j}}(t, \sigma(s))$ and integrating on $\left[t_{0}, t\right]_{\mathbb{T}}$, we get

$$
\begin{aligned}
& v_{j}(t) \\
& =v_{j}\left(t_{0}\right) e_{-b_{j}}\left(t, t_{0}\right) \\
& +\int_{t_{0}}^{t} e_{-b_{j}}(t, \sigma(s)) \\
& \times\left\{\sum _ { i = 1 } ^ { n } b _ { i j } ( s ) \left(h_{i}\left(x_{i}\left(s-\zeta_{i j}(s)\right)\right)\right.\right. \\
& +\sum_{i=1}^{n} q_{i j}(s)\left(k_{i}\left(x_{i}^{\Delta}\left(s-\varsigma_{i j}(s)\right)\right)\right. \\
& \left.\left.-k_{i}\left(\alpha_{i}^{\Delta}\left(s-\varsigma_{i j}(s)\right)\right)\right)\right\} \Delta s
\end{aligned}
$$

For positive constant $\alpha<\min \left\{\min _{1 \leq i \leq n} a_{i}^{-}, \min _{1 \leq j \leq m} b_{j}^{-}\right\}$with $-\alpha \in \mathscr{R}^{+}$, we have $e_{\ominus \alpha}\left(t, t_{0}\right)>1$, where $t \in\left[-\theta, t_{0}\right]_{\mathbb{T}}$. Take

$$
M>\max \left\{\epsilon_{1}, \epsilon_{2}\right\}
$$

where

$$
\begin{aligned}
& \epsilon_{1}=\max _{1 \leq i \leq n}\left\{\frac{a_{i}^{+} a_{i}^{-}}{a_{i}^{-}-\left(a_{i}^{+}+a_{i}^{-}\right) \varrho_{i}}, \frac{a_{i}^{-}}{a_{i}^{-}-\varrho_{i}}\right\}, \\
& \epsilon_{2}=\max _{1 \leq j \leq m}\left\{\frac{b_{j}^{+} b_{j}^{-}}{b_{j}^{-}-\left(b_{j}^{+}+b_{j}^{-}\right) \rho_{j}}, \frac{b_{j}^{-}}{b_{j}^{-}-\rho_{j}}\right\} .
\end{aligned}
$$

By $\left(\mathrm{H}_{4}\right)$, we have

$$
\begin{gathered}
a_{i}^{-}>\left(a_{i}^{+}+a_{i}^{-}\right) \varrho_{i}, \quad a_{i}^{-}>\varrho_{i}, \\
b_{j}^{-}>\left(b_{j}^{+}+b_{j}^{-}\right) \rho_{j}, \quad b_{j}^{-}>\rho_{j}, \\
i=1,2, \ldots, n, \quad j=1,2, \ldots, m .
\end{gathered}
$$

In view of $\left(\mathrm{H}_{5}\right)$, we have $M>1$. Hence, it is obvious that

$$
|v(t)|_{1} \leq M e_{\ominus \alpha}\left(t, t_{0}\right)\left\|\phi-\phi^{*}\right\|, \quad \forall t \in\left[-\theta, t_{0}\right]_{\mathbb{T}} \cdot
$$

We claim that

$$
|v(t)|_{1} \leq M e_{\ominus \alpha}\left(t, t_{0}\right)\left\|\phi-\phi^{*}\right\|, \quad \forall t \in\left(t_{0},+\infty\right)_{\mathbb{T}} .
$$

To prove this claim, we show that for any $p>1$, the following inequality holds:

$$
|v(t)|_{1}<p M e_{\ominus \alpha}\left(t, t_{0}\right)\left\|\phi-\phi^{*}\right\|, \quad \forall t \in\left(t_{0},+\infty\right)_{\mathbb{T}},
$$


which means that, for $i=1,2, \ldots, n$, we have

$$
\begin{array}{ll}
\left|u_{i}(t)\right|<p M e_{\ominus \alpha}\left(t, t_{0}\right)\left\|\phi-\phi^{*}\right\|, & \forall t \in\left(t_{0},+\infty\right)_{\mathbb{T}}, \\
\left|u_{i}^{\Delta}(t)\right|<p M e_{\ominus \alpha}\left(t, t_{0}\right)\left\|\phi-\phi^{*}\right\|, & \forall t \in\left(t_{0},+\infty\right)_{\mathbb{T}},
\end{array}
$$

and for $j=1,2, \ldots, m$, we have

$$
\begin{array}{ll}
\left|v_{j}(t)\right|<p M e_{\ominus \alpha}\left(t, t_{0}\right)\left\|\phi-\phi^{*}\right\|, & \forall t \in\left(t_{0},+\infty\right)_{\mathbb{T}}, \\
\left|v_{j}^{\Delta}(t)\right|<p M e_{\ominus \alpha}\left(t, t_{0}\right)\left\|\phi-\phi^{*}\right\|, & \forall t \in\left(t_{0},+\infty\right)_{\mathbb{T}} .
\end{array}
$$

By way of contradiction, assume that (44) does not hold. Firstly, we consider the following four cases.

Case 1. Equation (45) is not true and (46)-(48) are all true. Then, there exists $t_{1} \in\left(t_{0},+\infty\right)_{\mathbb{V}}$ and $i_{0} \in\{1,2, \ldots, n\}$ such that

$$
\begin{gathered}
\left|u_{i_{0}}\left(t_{1}\right)\right| \geq p M e_{\ominus \alpha}\left(t_{1}, t_{0}\right)\left\|\phi-\phi^{*}\right\|, \\
\left|u_{i_{0}}(t)\right|<p M e_{\ominus \alpha}\left(t, t_{0}\right)\left\|\phi-\phi^{*}\right\|, \quad t \in\left(t_{0}, t_{1}\right)_{\mathbb{T}}, \\
\quad\left|u_{l}(t)\right|<p M e_{\ominus \alpha}\left(t, t_{0}\right)\left\|\phi-\phi^{*}\right\|, \\
\text { for } l \neq i_{0}, \quad t \in\left(t_{0}, t_{1}\right]_{\mathbb{T}}, \quad l=1,2, \ldots, n .
\end{gathered}
$$

Therefore, there must be a constant $\delta_{1} \geq 1$ such that

$$
\begin{gathered}
\left|u_{i_{0}}\left(t_{1}\right)\right|=\delta_{1} p M e_{\ominus \alpha}\left(t_{1}, t_{0}\right)\left\|\phi-\phi^{*}\right\|, \\
\left|u_{i_{0}}(t)\right|<\delta_{1} p M e_{\ominus \alpha}\left(t, t_{0}\right)\left\|\phi-\phi^{*}\right\|, \quad t \in\left(t_{0}, t_{1}\right)_{\mathbb{T}}, \\
\left|u_{l}(t)\right|<\delta_{1} p M e_{\ominus \alpha}\left(t, t_{0}\right)\left\|\phi-\phi^{*}\right\|, \\
\text { for } l \neq i_{0}, \quad t \in\left(t_{0}, t_{1}\right]_{\mathbb{T}}, \quad l=1,2, \ldots, n .
\end{gathered}
$$

Note that, in view of (37), we have

$$
\begin{aligned}
& \left|u_{i_{0}}\left(t_{1}\right)\right| \\
& =\mid u_{i_{0}}\left(t_{0}\right) e_{-a_{i_{0}}}\left(t_{1}, t_{0}\right)+\int_{t_{0}}^{t_{1}} e_{-a_{i_{0}}}\left(t_{1}, \sigma(s)\right)
\end{aligned}
$$

$$
\begin{aligned}
& \times\left\{\sum _ { j = 1 } ^ { m } a _ { j i _ { 0 } } ( s ) \left(f_{j}\left(y_{j}\left(s-\tau_{j i_{0}}(s)\right)\right)\right.\right. \\
& \left.-f_{j}\left(\beta_{j}\left(s-\tau_{j i_{0}}(s)\right)\right)\right) \\
& +\sum_{j=1}^{m} p_{j i_{0}}(s)\left(g_{j}\left(y_{j}^{\Delta}\left(s-\sigma_{j i_{0}}(s)\right)\right)\right. \\
& \left.\left.-g_{j}\left(\beta_{j}^{\Delta}\left(s-\sigma_{j i_{0}}(s)\right)\right)\right)\right\} \Delta s \\
& \leq e_{-a_{i_{0}}}\left(t_{1}, t_{0}\right)\left\|\phi-\phi^{*}\right\| \\
& +\int_{t_{0}}^{t_{1}} e_{-a_{i_{0}}}\left(t_{1}, \sigma(s)\right) \\
& \times\left(\sum_{j=1}^{m} a_{j i_{0}}^{+} H_{j}^{f}\left|v_{j}\left(s-\tau_{j i_{0}}(s)\right)\right|\right. \\
& \left.+\sum_{j=1}^{n} p_{j i_{0}}^{+} H_{j}^{g}\left|v_{j}^{\Delta}\left(s-\sigma_{j i_{0}}(s)\right)\right|\right) \Delta s \\
& \leq e_{-a_{i_{0}}}\left(t_{1}, t_{0}\right)\left\|\phi-\phi^{*}\right\| \\
& +\int_{t_{0}}^{t_{1}} e_{-a_{i_{0}}}\left(t_{1}, \sigma(s)\right) \\
& \times\left(\sum_{j=1}^{m} a_{j i_{0}}^{+} H_{j}^{f} \delta_{1} p M e_{\ominus \alpha}\left(s-\tau_{j i_{0}}(s), t_{0}\right)\left\|\phi-\phi^{*}\right\|\right. \\
& \left.+\sum_{j=1}^{m} p_{j i_{0}}^{+} H_{j}^{g} \delta_{1} p M e_{\ominus \alpha}\left(s-\sigma_{j i_{0}}(s), t_{0}\right)\left\|\phi-\phi^{*}\right\|\right) \Delta s \\
& =\left\|\phi-\phi^{*}\right\| e_{\ominus \alpha}\left(t_{1}, t_{0}\right) e_{-a_{i_{0}} \oplus \alpha}\left(t_{1}, t_{0}\right) \\
& +\delta_{1} p M e_{\ominus \alpha}\left(t_{1}, t_{0}\right)\left\|\phi-\phi^{*}\right\| \\
& \times \int_{t_{0}}^{t_{1}} e_{-a_{i_{0}}}\left(t_{1}, \sigma(s)\right) \\
& \times\left(\sum_{j=1}^{m} a_{j i_{0}}^{+} H_{j}^{f} e_{\ominus \alpha}\left(s-\tau_{j i_{0}}(s), t_{1}\right)\right. \\
& \left.+\sum_{j=1}^{m} p_{j i_{0}}^{+} H_{j}^{g} e_{\ominus \alpha}\left(s-\sigma_{j i_{0}}(s), t_{1}\right)\right) \Delta s \\
& \leq\left\|\phi-\phi^{*}\right\| e_{\ominus \alpha}\left(t_{1}, t_{0}\right) e_{-a_{i_{0}}^{-}+\alpha}\left(t_{1}, t_{0}\right) \\
& +\delta_{1} p M e_{\ominus \alpha}\left(t_{1}, t_{0}\right)\left\|\phi-\phi^{*}\right\| \\
& \times \int_{t_{0}}^{t_{1}} e_{-a_{i_{0}}^{-}}\left(t_{1}, \sigma(s)\right)
\end{aligned}
$$


Journal of Applied Mathematics

9

$$
\begin{aligned}
& \times\left(\sum_{j=1}^{m} a_{j i_{0}}^{+} H_{j}^{f} \exp \{-\alpha \widetilde{\tau}\}+\sum_{j=1}^{m} p_{j i_{0}}^{+} H_{j}^{g} \exp \{-\alpha \widetilde{\sigma}\}\right) \Delta s \\
& \leq\left\|\phi-\phi^{*}\right\| e_{\ominus \alpha}\left(t_{1}, t_{0}\right)+\delta_{1} p M e_{\ominus \alpha}\left(t_{1}, t_{0}\right)\left\|\phi-\phi^{*}\right\| \frac{1}{-a_{i_{0}}^{-}} \\
& \times \int_{t_{0}}^{t_{1}}\left(-a_{i_{0}}^{-}\right) e_{-a_{i_{0}}^{-}}\left(t_{1}, \sigma(s)\right) \Delta s \\
& \times\left(\sum_{j=1}^{m} a_{j i_{0}}^{+} H_{j}^{f} \exp \{-\alpha \widetilde{\tau}\}+\sum_{j=1}^{m} p_{j i_{0}}^{+} H_{j}^{g} \exp \{-\alpha \widetilde{\sigma}\}\right) \\
& =\left\|\phi-\phi^{*}\right\| e_{\ominus \alpha}\left(t_{1}, t_{0}\right)-\frac{1}{a_{i_{0}}^{-}} \delta_{1} p M e_{\ominus(-\alpha)}\left(t_{1}, t_{0}\right) \\
& \times\left\|\phi-\phi^{*}\right\|\left(e_{-a_{i_{0}}^{-}}\left(t_{1}, t_{0}\right)-1\right) \\
& \times\left(\sum_{j=1}^{m} a_{j i_{0}}^{+} H_{j}^{f} \exp \{-\alpha \widetilde{\tau}\}+\sum_{j=1}^{m} p_{j i_{0}}^{+} H_{j}^{g} \exp \{-\alpha \widetilde{\sigma}\}\right) \\
& <\delta_{1} p M\left\|\phi-\phi^{*}\right\| e_{\ominus \alpha}\left(t_{1}, t_{0}\right) \\
& \times\left[\frac{1}{\delta_{1} p M}+\frac{1}{a_{i_{0}}^{-}}\left(\sum_{j=1}^{m} a_{j i_{0}}^{+} H_{j}^{f} \exp \{-\alpha \tilde{\tau}\}\right.\right. \\
& \left.\left.+\sum_{j=1}^{m} p_{j i_{0}}^{+} H_{j}^{g} \exp \{-\alpha \widetilde{\sigma}\}\right)\right] \\
& <\delta_{1} p M\left\|\phi-\phi^{*}\right\| e_{\ominus \alpha}\left(t_{1}, t_{0}\right) \\
& \times\left(\frac{1}{M}+\frac{1}{a_{i_{0}}^{-}} \sum_{j=1}^{m}\left(a_{j i_{0}}^{+} H_{j}^{f}+p_{j i_{0}}^{+} H_{j}^{g}\right)\right) \\
& <\delta_{1} p M e_{\ominus \alpha}\left(t_{1}, t_{0}\right)\left\|\phi-\phi^{*}\right\|,
\end{aligned}
$$

where $\tilde{\tau}=\min _{(i, j)} \tau_{j i}^{-}, \widetilde{\sigma}=\min _{(i, j)} \sigma_{j i}^{-}$. In the proof, we use the inequality $e_{-a_{i}^{-}}\left(t_{1}, t_{0}\right)<1$. Thus, we get a contradiction.

Case 2. Equation (46) is not true and (45), (47), and (48) are all true. Then, there exists $t_{2} \in\left(t_{0},+\infty\right)_{\mathbb{T}}$ and $i_{1} \in\{1,2, \ldots, n\}$ such that

$$
\begin{gathered}
\left|u_{i_{1}}^{\Delta}\left(t_{2}\right)\right| \geq p M e_{\ominus \alpha}\left(t_{2}, t_{0}\right)\left\|\phi-\phi^{*}\right\|, \\
\left|u_{i_{1}}^{\Delta}(t)\right|<p M e_{\ominus \alpha}\left(t, t_{0}\right)\left\|\phi-\phi^{*}\right\|, \quad t \in\left(t_{0}, t_{2}\right)_{\mathbb{T}} \\
\left|u_{l}^{\Delta}(t)\right|<p M e_{\ominus \alpha}\left(t, t_{0}\right)\left\|\phi-\phi^{*}\right\| \\
\text { for } l \neq i_{1}, t \in\left(t_{0}, t_{2}\right]_{\mathbb{T}}, \quad l=1,2, \ldots, n .
\end{gathered}
$$

Hence, there must be a constant $\delta_{2} \geq 1$ such that

$$
\begin{gathered}
\left|u_{i_{1}}^{\Delta}\left(t_{2}\right)\right|=\delta_{2} p M e_{\ominus \alpha}\left(t_{2}, t_{0}\right)\left\|\phi-\phi^{*}\right\|, \\
\left|u_{i_{1}}^{\Delta}(t)\right|<\delta_{2} p M e_{\ominus \alpha}\left(t, t_{0}\right)\left\|\phi-\phi^{*}\right\|, \quad t \in\left(t_{0}, t_{2}\right)_{\mathbb{T}}, \\
\quad\left|u_{l}^{\Delta}(t)\right|<\delta_{2} p M e_{\ominus \alpha}\left(t, t_{0}\right)\left\|\phi-\phi^{*}\right\|, \\
\text { for } l \neq i_{1}, \quad t \in\left(t_{0}, t_{2}\right]_{\mathbb{T}}, \quad l=1,2, \ldots, n .
\end{gathered}
$$

Note that, in view of (37), we have

$$
\left.\begin{array}{c}
u_{i}^{\Delta}(t) \\
=-a_{i}(t) u_{i}\left(t_{0}\right) e_{-a_{i}}\left(t, t_{0}\right) \\
+\int_{t_{0}}^{t}\left(-a_{i}(t)\right) e_{-a_{i}}(t, \sigma(s)) \\
\times\left\{\sum _ { j = 1 } ^ { m } a _ { j i } ( s ) \left(f_{j}\left(y_{j}\left(s-\tau_{j i}(s)\right)\right)\right.\right. \\
\left.-f_{j}\left(\beta_{j}\left(s-\tau_{j i}(s)\right)\right)\right) \\
+\sum_{j=1}^{m} p_{j i}(s)\left(g_{j}\left(y_{j}^{\Delta}\left(s-\sigma_{j i}(s)\right)\right)\right. \\
+\sum_{j=1}^{m} p_{j i}(t)\left(g_{j}\left(y_{j}^{\Delta}\left(t-\sigma_{j i}(t)\right)\right)\right. \\
\times\left\{\sum _ { j = 1 } ^ { m } a _ { j i } ( t ) \left(f_{j}\left(y_{j}\left(t-\tau_{j i}(t)\right)\right)\right.\right. \\
-\sigma(t)) \\
\left.-f_{j}\left(\beta_{j}\left(t-\tau_{j i}(t)\right)\right)\right)
\end{array}\right\} \Delta s
$$

Thus, we have

$$
\begin{aligned}
& \left|u_{i_{1}}^{\Delta}\left(t_{2}\right)\right| \\
& \leq a_{i_{1}}^{+} e_{-a_{i_{1}}^{-}}\left(t_{2}, t_{0}\right)\left\|\phi-\phi^{*}\right\|
\end{aligned}
$$




$$
\begin{aligned}
& +a_{i_{1}}^{+} \int_{t_{0}}^{t_{2}} e_{-a_{i_{1}}}\left(t_{2}, \sigma(s)\right) \\
& \times\left(\sum_{j=1}^{m} a_{j i_{1}}^{+} H_{j}^{f}\left|\nu_{j}\left(s-\tau_{j i_{1}}(s)\right)\right|\right. \\
& \left.+\sum_{j=1}^{m} p_{j i_{1}}^{+} H_{j}^{g}\left|v_{j}^{\Delta}\left(s-\sigma_{j i_{1}}(s)\right)\right|\right) \Delta s \\
& +\sum_{j=1}^{m} a_{j i_{1}}^{+} H_{j}^{f}\left|v_{j}\left(t_{2}-\tau_{j i_{1}}\left(t_{2}\right)\right)\right| \\
& +\sum_{j=1}^{m} p_{j i_{1}}^{+} H_{j}^{g}\left|v_{j}^{\Delta}\left(t_{2}-\sigma_{j i_{1}}\left(t_{2}\right)\right)\right| \\
& \leq a_{i_{1}}^{+} e_{-a_{i_{1}}^{-}}\left(t_{2}, t_{0}\right)\left\|\phi-\phi^{*}\right\| \\
& +a_{i_{1}}^{+} \int_{t_{0}}^{t_{2}} e_{-a_{i_{1}}}\left(t_{2}, \sigma(s)\right) \\
& \times\left(\sum_{j=1}^{m} a_{j i_{1}}^{+} H_{j}^{f} \delta_{2} p M e_{\ominus(-\alpha)}\left(s-\tau_{j i_{1}}(s), t_{0}\right)\left\|\phi-\phi^{*}\right\|\right. \\
& +\sum_{j=1}^{m} p_{j i_{1}}^{+} H_{j}^{g} \delta_{2} p M e_{\ominus \alpha}\left(s-\sigma_{j i_{1}}(s), t_{0}\right) \\
& \left.\times\left\|\phi-\phi^{*}\right\|\right) \Delta s \\
& +\delta_{2} p M e_{\ominus \alpha}\left(t_{2}-\tau_{j i_{1}}\left(t_{2}\right), t_{0}\right)\left\|\phi-\phi^{*}\right\| \sum_{j=1}^{m} a_{j i_{1}}^{+} H_{j}^{f} \\
& +\delta_{2} p M e_{\ominus \alpha}\left(t_{2}-\sigma_{j i_{1}}\left(t_{2}\right), t_{0}\right)\left\|\phi-\phi^{*}\right\| \sum_{j=1}^{m} p_{j i_{1}}^{+} H_{j}^{g} \\
& \leq a_{i_{1}}^{+} e_{-a_{i_{1}}^{-}}\left(t_{2}, t_{0}\right)\left\|\phi-\phi^{*}\right\| \\
& +a_{i_{1}}^{+} \delta_{2} p M e_{\ominus \alpha}\left(t_{2}, t_{0}\right)\left\|\phi-\phi^{*}\right\| \\
& \times \int_{t_{0}}^{t_{2}} e_{-a_{i_{1}}}\left(t_{2}, \sigma(s)\right) \\
& \times\left(\sum_{j=1}^{m} a_{j i_{1}}^{+} H_{j}^{f} e_{\ominus(-\alpha)}\left(s-\tau_{j i_{1}}(s), t_{2}\right)\right. \\
& \left.+\sum_{j=1}^{m} p_{j i_{1}}^{+} H_{j}^{g} e_{\ominus \alpha}\left(s-\sigma_{j i_{1}}(s), t_{2}\right)\right) \Delta s \\
& +\delta_{2} p M e_{\ominus \alpha}\left(t_{2}, t_{0}\right)\left\|\phi-\phi^{*}\right\| \\
& \times \sum_{j=1}^{m}\left(a_{j i_{1}}^{+} H_{j}^{f} e_{\ominus \alpha}\left(t_{2}-\tau_{j i_{1}}\left(t_{2}\right), t_{2}\right)\right. \\
& \left.+p_{j i_{1}}^{+} H_{j}^{g} e_{\ominus \alpha}\left(t_{2}-\sigma_{j i_{1}}\left(t_{2}\right), t_{2}\right)\right) \\
& \leq a_{i_{1}}^{+} e_{-a_{i_{1}}^{-}}\left(t_{2}, t_{0}\right)\left\|\phi-\phi^{*}\right\|
\end{aligned}
$$

$$
\begin{aligned}
& +a_{i_{1}}^{+} \delta_{2} p M e_{\ominus \alpha}\left(t_{2}, t_{0}\right)\left\|\phi-\phi^{*}\right\| \\
& \times \int_{t_{0}}^{t_{2}} e_{-a_{i_{1}}^{-}}\left(t_{2}, \sigma(s)\right) \\
& \times\left(\sum_{j=1}^{m} a_{j i_{1}}^{+} H_{j}^{f} \exp \{-\alpha \widetilde{\tau}\}+\sum_{j=1}^{m} p_{j i_{1}}^{+} H_{j}^{g} \exp \{-\alpha \widetilde{\sigma}\}\right) \Delta s \\
& +\delta_{2} p M e_{\ominus \alpha}\left(t_{2}, t_{0}\right)\left\|\phi-\phi^{*}\right\| \\
& \times \sum_{j=1}^{m}\left(a_{j i_{1}}^{+} H_{j}^{f} \exp \{-\alpha \tilde{\tau}\}+p_{j i_{1}}^{+} H_{j}^{g} \exp \{-\alpha \widetilde{\sigma}\}\right) \\
& \leq a_{i_{1}}^{+} e_{-a_{i_{1}}^{-}}\left(t_{2}, t_{0}\right)\left\|\phi-\phi^{*}\right\|+a_{i_{1}}^{+} \delta_{2} p M e_{\ominus \alpha}\left(t_{2}, t_{0}\right)\left\|\phi-\phi^{*}\right\| \\
& \times \frac{1}{-a_{i_{1}}^{-}} \int_{t_{0}}^{t_{2}}\left(-a_{i_{1}}^{-}\right) e_{-a_{i_{1}}^{-}}\left(t_{2}, \sigma(s)\right) \Delta s \\
& \times\left(\sum_{j=1}^{m} a_{j i_{1}}^{+} H_{j}^{f} \exp \{-\alpha \widetilde{\tau}\}+\sum_{j=1}^{m} p_{j i_{1}}^{+} H_{j}^{g} \exp \{-\alpha \widetilde{\sigma}\}\right) \\
& +\delta_{2} p M e_{\ominus \alpha}\left(t_{2}, t_{0}\right)\left\|\phi-\phi^{*}\right\| \sum_{j=1}^{m}\left(a_{j i_{1}}^{+} H_{j}^{f}+p_{j i_{1}}^{+} H_{j}^{g}\right) \\
& =a_{i_{1}}^{+}\left\|\phi-\phi^{*}\right\| e_{\ominus \alpha}\left(t_{2}, t_{0}\right) e_{-a_{i_{1}}^{-} \oplus \alpha}\left(t_{2}, t_{0}\right) \\
& -\frac{a_{i_{1}}^{+}}{a_{i_{1}}^{-}} \delta_{2} p M e_{\ominus \alpha}\left(t_{2}, t_{0}\right)\left\|\phi-\phi^{*}\right\|\left(e_{-a_{i_{1}}^{-}}\left(t_{2}, t_{0}\right)-1\right) \\
& \times\left(\sum_{j=1}^{m} a_{j i_{1}}^{+} H_{j}^{f} \times \exp \{-\alpha \widetilde{\tau}\}+\sum_{j=1}^{m} p_{j i_{1}}^{+} H_{j}^{g} \exp \{-\alpha \widetilde{\sigma}\}\right) \\
& +\delta_{2} p M e_{\ominus \alpha}\left(t_{2}, t_{0}\right)\left\|\phi-\phi^{*}\right\| \sum_{j=1}^{m}\left(a_{j i_{1}}^{+} H_{j}^{f}+p_{j i_{1}}^{+} H_{j}^{g}\right) \\
& \leq \delta_{2} p M e_{\ominus \alpha}\left(t_{2}, t_{0}\right)\left\|\phi-\phi^{*}\right\| \\
& \times\left\{\frac{a_{i_{1}}^{+}}{\delta_{2} p M}+\sum_{j=1}^{m}\left(a_{j i_{1}}^{+} H_{j}^{f}+p_{j i_{1}}^{+} H_{j}^{g}\right)\right. \\
& +\frac{a_{i_{1}}^{+}}{a_{i_{1}}^{-}} \sum_{j=1}^{m}\left(a_{j i_{1}}^{+} H_{j}^{f} \exp \{-\alpha \tilde{\tau}\}\right. \\
& \left.\left.+p_{j i_{1}}^{+} H_{j}^{g} \exp \{-\alpha \widetilde{\sigma}\}\right)\right\} \\
& <\delta_{2} p M e_{\ominus \alpha}\left(t_{2}, t_{0}\right)\left\|\phi-\phi^{*}\right\| \\
& \times\left\{\frac{a_{i_{1}}^{+}}{M}+\left(1+\frac{a_{i_{1}}^{+}}{a_{i_{1}}^{-}}\right) \sum_{j=1}^{m}\left(a_{j i_{1}}^{+} H_{j}^{f}+p_{j i_{1}}^{+} H_{j}^{g}\right)\right\} \\
& <\delta_{2} p M e_{\ominus \alpha}\left(t_{2}, t_{0}\right)\left\|\phi-\phi^{*}\right\|
\end{aligned}
$$

We also get a contradiction. 
Case 3. Equation (47) is not true and (45), (46), and (48) are all true. Then, there exists $t_{3} \in\left(t_{0},+\infty\right)_{\mathbb{T}}$ and $j_{0} \in$ $\{1,2, \ldots, m\}$ such that

$$
\begin{gathered}
\left|v_{j_{0}}\left(t_{3}\right)\right| \geq p M e_{\ominus \alpha}\left(t_{3}, t_{0}\right)\left\|\phi-\phi^{*}\right\|, \\
\left|v_{j_{0}}(t)\right|<p M e_{\ominus \alpha}\left(t, t_{0}\right)\left\|\phi-\phi^{*}\right\|, \quad t \in\left(t_{0}, t_{3}\right)_{\mathbb{T}}, \\
\left|v_{\iota}(t)\right|<p M e_{\ominus \alpha}\left(t, t_{0}\right)\left\|\phi-\phi^{*}\right\|,
\end{gathered}
$$$$
\text { for } \iota \neq j_{0}, t \in\left(t_{0}, t_{3}\right]_{\mathbb{T}}, \iota=1,2, \ldots, m \text {. }
$$

Therefore, there must be a constant $\delta_{3} \geq 1$ such that

$$
\begin{gathered}
\left|v_{j_{0}}\left(t_{3}\right)\right|=\delta_{3} p M e_{\ominus \alpha}\left(t_{3}, t_{0}\right)\left\|\phi-\phi^{*}\right\|, \\
\left|v_{j_{0}}(t)\right|<\delta_{3} p M e_{\ominus \alpha}\left(t, t_{0}\right)\left\|\phi-\phi^{*}\right\|, \quad t \in\left(t_{0}, t_{3}\right)_{\mathbb{T}}, \\
\quad\left|\nu_{\iota}(t)\right|<\delta_{3} p M e_{\ominus \alpha}\left(t, t_{0}\right)\left\|\phi-\phi^{*}\right\|, \\
\text { for } \iota \neq j_{0}, t \in\left(t_{0}, t_{3}\right]_{\mathbb{T}}, \quad \iota=1,2, \ldots, m .
\end{gathered}
$$

Then, in a similar way, in view of (38), we have that

$$
\begin{aligned}
\left|v_{j_{0}}\left(t_{3}\right)\right|< & \delta_{3} p M\left\|\phi-\phi^{*}\right\| e_{\ominus \alpha}\left(t_{3}, t_{0}\right) \\
& \times\left(\frac{1}{M}+\frac{1}{b_{j_{0}}^{-}} \sum_{i=1}^{n}\left(b_{i j_{0}}^{+} H_{i}^{h}+q_{i j_{0}}^{+} H_{i}^{k}\right)\right) \\
< & \delta_{3} p M e_{\ominus \alpha}\left(t_{3}, t_{0}\right)\left\|\phi-\phi^{*}\right\|,
\end{aligned}
$$

which is also a contradiction.

Case 4. Equation (48) is not true and (45), (46), and (47) are all true. Then, there exists $t_{4} \in\left(t_{0},+\infty\right)_{\mathbb{T}}$ and $j_{1} \in$ $\{1,2, \ldots, m\}$ such that

$$
\begin{gathered}
\left|v_{j_{1}}^{\Delta}\left(t_{4}\right)\right| \geq p M e_{\ominus \alpha}\left(t_{4}, t_{0}\right)\left\|\phi-\phi^{*}\right\|, \\
\left|v_{j_{1}}^{\Delta}(t)\right|<p M e_{\ominus \alpha}\left(t, t_{0}\right)\left\|\phi-\phi^{*}\right\|, \quad t \in\left(t_{0}, t_{4}\right)_{\mathbb{T}}, \\
\left|v_{\iota}^{\Delta}(t)\right|<p M e_{\ominus \alpha}\left(t, t_{0}\right)\left\|\phi-\phi^{*}\right\|,
\end{gathered}
$$

for $\iota \neq j_{1}, t \in\left(t_{0}, t_{4}\right]_{\mathbb{T}}, \iota=1,2, \ldots, m$.

Hence, there must be a constant $\delta_{4} \geq 1$ such that

$$
\begin{gathered}
\left|v_{j_{1}}^{\Delta}\left(t_{4}\right)\right| \geq \delta_{4} p M e_{\ominus \alpha}\left(t_{4}, t_{0}\right)\left\|\phi-\phi^{*}\right\|, \\
\left|v_{j_{1}}^{\Delta}(t)\right|<\delta_{4} p M e_{\ominus \alpha}\left(t, t_{0}\right)\left\|\phi-\phi^{*}\right\|, \quad t \in\left(t_{0}, t_{4}\right)_{\mathbb{T}}, \\
\left|v_{\iota}^{\Delta}(t)\right|<\delta_{4} p M e_{\ominus \alpha}\left(t, t_{0}\right)\left\|\phi-\phi^{*}\right\|, \\
\text { for } \iota \neq j_{1}, t \in\left(t_{0}, t_{4}\right]_{\mathbb{T}}, \iota=1,2, \ldots, m .
\end{gathered}
$$

Similarly, in view of (38), we obtain that

$$
\begin{aligned}
\left|v_{j_{1}}^{\Delta}\left(t_{4}\right)\right|< & \delta_{4} p M e_{\ominus \alpha}\left(t_{4}, t_{0}\right)\left\|\phi-\phi^{*}\right\| \\
& \times\left\{\frac{b_{j_{1}}^{+}}{M}+\left(1+\frac{b_{j_{1}}^{+}}{b_{j_{1}}^{-}}\right) \sum_{i=1}^{n}\left(b_{i j_{0}}^{+} H_{i}^{h}+q_{i j_{0}}^{+} H_{i}^{k}\right)\right\} \\
< & \delta_{4} p M e_{\ominus \alpha}\left(t_{4}, t_{0}\right)\left\|\phi-\phi^{*}\right\| .
\end{aligned}
$$

It is also a contradiction.
By the above four cases, for other cases of negative proposition of (44), we can obtain a contradiction. Therefore, (44) holds. Let $p \rightarrow 1$, then (43) holds. We can take $-\lambda=\ominus \alpha$, then $\lambda>0$ and $-\lambda \in \mathscr{R}^{+}$. Hence, we have that

$$
|v(t)|_{1} \leq M\left\|\phi-\phi^{*}\right\| e_{-\lambda}\left(t, t_{0}\right), \quad t \in[-\theta, \infty)_{\mathbb{T}}, t \geq t_{0},
$$

which means that the almost periodic solution $\omega(t)$ of $(2)$ is exponentially stable. This completes the proof of Theorem 18.

\section{An Example}

In this section, we present an example to illustrate the feasibility of our results obtained in previous sections.

Example 1. Let $n=m=2$. Consider the following neutraltype BAM neural networks with delays on a time scale $\mathbb{T}$

$$
\begin{aligned}
x_{i}^{\Delta}(t)= & -a_{i}(t) x_{i}(t)+\sum_{j=1}^{2} a_{j i}(t) f_{j}\left(y_{j}\left(t-\tau_{j i}(t)\right)\right) \\
& +\sum_{j=1}^{2} p_{j i}(t) g_{j}\left(y_{j}^{\Delta}\left(t-\sigma_{j i}(t)\right)\right)+I_{i}(t), \\
y_{j}^{\Delta}(t)= & -b_{j}(t) y_{j}(t)+\sum_{i=1}^{2} b_{i j}(t) h_{i}\left(x_{i}\left(t-\zeta_{i j}(t)\right)\right) \\
& +\sum_{i=1}^{2} q_{i j}(t) k_{i}\left(x_{i}^{\Delta}\left(t-\varsigma_{i j}(t)\right)\right)+J_{j}(t),
\end{aligned}
$$

where $t \in \mathbb{T}, \quad i, j=1,2$ and the coefficients are as follows:

$$
\begin{gathered}
a_{1}(t)=0.975+0.025 \sin t, \quad a_{2}(t)=0.93+0.01 \cos t, \\
a_{11}(t)=0.003 \sin t, \quad a_{12}(t)=0.002 \cos t, \\
a_{21}(t)=0.004 \sin t, \quad a_{22}(t)=0.001 \sin t, \\
p_{11}(t)=0.001+0.003 \cos t, \quad p_{12}(t)=0.001 \sin t, \\
p_{21}(t)=0.006+0.001 \sin t, \quad p_{22}(t)=0.003 \cos t, \\
f_{1}(u)=0.2 \sin u, \quad f_{2}(u)=2 \cos u, \\
g_{1}(u)=0.2 \cos u, \quad g_{2}(u)=2 \sin u, \\
b_{1}(t)=0.95+0.01 \sin \sqrt{3} t, \quad b_{2}(t)=0.92+0.01 \cos \sqrt{2} t, \\
b_{11}(t)=0.002 \sin \sqrt{2} t, \quad b_{12}(t)=0.003 \cos t, \\
b_{21}(t)=0.001 \sin t, \quad b_{22}(t)=0.004 \cos t,
\end{gathered}
$$




$$
\begin{gathered}
q_{11}(t)=0.001 \cos \sqrt{3} t, \quad q_{12}(t)=0.004 \sin t, \\
q_{21}(t)=0.003 \sin t, \quad q_{22}(t)=0.004 \cos t, \\
h_{1}(u)=\sin u, \quad h_{2}(u)=1.5 \cos u, \\
k_{1}(u)=\cos u, \quad k_{2}(u)=1.5 \sin u, \\
I_{1}(t)=I_{2}(t)=0.6 \sin \sqrt{3} t, \\
J_{1}(t)=J_{2}(t)=0.5 \cos t, \quad \tau_{j i}(t)=\sigma_{j i}(t)=0.12 \sin t, \\
\zeta_{i j}(t)=\zeta_{i j}(t)=0.37 \sin t, \quad i, j=1,2 .
\end{gathered}
$$

By calculating, we have

$$
\begin{array}{cl}
a_{1}^{+}=1, & a_{1}^{-}=0.95, \\
a_{2}^{+}=0.94, & a_{2}^{-}=0.92, \\
b_{1}^{+}=0.96, & b_{1}^{-}=0.94, \\
b_{2}^{+}=0.93, & b_{2}^{-}=0.91, \\
a_{11}^{+}=0.003, & a_{12}^{+}=0.002, \\
a_{21}^{+}=0.004, & a_{22}^{+}=0.001, \\
p_{11}^{+}=0.004, & p_{12}^{+}=0.001, \\
p_{21}^{+}=0.007, & p_{22}^{+}=0.003, \\
b_{11}^{+}=0.002, & b_{12}^{+}=0.003, \\
b_{21}^{+}=0.001, & b_{22}^{+}=0.004, \\
q_{11}^{+}=0.001, & q_{12}^{+}=0.004, \\
q_{21}^{+}=0.003, & q_{22}^{+}=0.004, \\
H_{1}^{f}=H_{1}^{g}=0.2, & H_{2}^{f}=H_{2}^{g}=2, \\
H_{1}^{h}=H_{1}^{k}=1, & H_{2}^{h}=H_{2}^{k}=1.5 .
\end{array}
$$

If $\mathbb{T}=\mathbb{R}$, then $\mu(t)=0$, and if $\mathbb{T}=\mathbb{Z}$, then $\mu(t)=1$. We can verify for the above two cases, and all conditions of Theorems 17 and 18 are satisfied. Therefore, whether $\mathbb{T}=\mathbb{R}$ or $\mathbb{T}=\mathbb{Z}$, (63) has an almost periodic solution, which is exponentially stable. That is, the continuous-time neural network and its discrete-time analogue have the same dynamical behaviors.

\section{Conclusion}

In this paper, we establish some sufficient conditions ensuring the existence and exponential stability of almost periodic solutions for a class of neutral-type BAM neural networks with delays on time scales. Our results obtained in this paper are completely new even in case of the time scale $\mathbb{T}=\mathbb{R}$ or $\mathbb{Z}$ and complementary to the previously known results. Besides, our method used in this paper may be used to study many other neutral-type neural networks with delays such as shunting inhibitory cellular neural networks, CohenGrossberg neural networks, and so on.

\section{Acknowledgment}

This work is supported by the National Natural Sciences Foundation of People's Republic of China under Grant 10971183.

\section{References}

[1] B. Kosko, "Bidirectional associative memories," IEEE Transactions on Systems, Man, and Cybernetics, vol. 18, no. 1, pp. 49-60, 1988.

[2] Y. Li, "Global exponential stability of BAM neural networks with delays and impulses," Chaos, Solitons \& Fractals, vol. 24, no. 1, pp. 279-285, 2005.

[3] H. Zhao, "Global stability of bidirectional associative memory neural networks with distributed delays," Physics Letters A, vol. 297, no. 3-4, pp. 182-190, 2002.

[4] Q. Zhou, "Global exponential stability of BAM neural networks with distributed delays and impulses," Nonlinear Analysis: Real World Applications, vol. 10, no. 1, pp. 144-153, 2009.

[5] Y. Li, "Existence and stability of periodic solution for BAM neural networks with distributed delays," Applied Mathematics and Computation, vol. 159, no. 3, pp. 847-862, 2004.

[6] B. Zheng, Y. Zhang, and C. Zhang, "Global existence of periodic solutions on a simplified BAM neural network model with delays," Chaos, Solitons \& Fractals, vol. 37, no. 5, pp. 1397-1408, 2008.

[7] Y. Xia, J. Cao, and M. Lin, "New results on the existence and uniqueness of almost periodic solution for BAM neural networks with continuously distributed delays," Chaos, Solitons \& Fractals, vol. 31, no. 4, pp. 928-936, 2007.

[8] L. Zhang and L. Si, "Existence and exponential stability of almost periodic solution for BAM neural networks with variable coefficients and delays," Applied Mathematics and Computation, vol. 194, no. 1, pp. 215-223, 2007.

[9] A. Chen, L. Huang, and J. Cao, "Existence and stability of almost periodic solution for BAM neural networks with delays," Applied Mathematics and Computation, vol. 137, no. 1, pp. 177193, 2003.

[10] Y. Li and X. Fan, "Existence and globally exponential stability of almost periodic solution for Cohen-Grossberg BAM neural networks with variable coefficients," Applied Mathematical Modelling, vol. 33, no. 4, pp. 2114-2120, 2009.

[11] C. Liu, C. Li, and X. Liao, "Variable-time impulses in BAM neural networks with delays," Neurocomputing, vol. 74, no. 17, pp. 3286-3295, 2011.

[12] Z. Zhang and K. Liu, "Existence and global exponential stability of a periodic solution to interval general bidirectional associative memory (BAM) neural networks with multiple delays on time scales," Neural Networks, vol. 24, no. 5, pp. 427-439, 2011.

[13] Y. Li and S. Gao, "Global exponential stability for impulsive BAM neural networks with distributed delays on time scales," Neural Processing Letters, vol. 31, no. 1, pp. 65-91, 2010.

[14] J. H. Park, C. H. Park, O. M. Kwon, and S. M. Lee, "A new stability criterion for bidirectional associative memory neural networks of neutral-type," Applied Mathematics and Computation, vol. 199, no. 2, pp. 716-722, 2008. 
[15] R. Rakkiyappan and P. Balasubramaniam, "New global exponential stability results for neutral type neural networks with distributed time delays," Neurocomputing, vol. 71, no. 4-6, pp. 1039-1045, 2008.

[16] R. Rakkiyappan and P. Balasubramaniam, "LMI conditions for global asymptotic stability results for neutral-type neural networks with distributed time delays," Applied Mathematics and Computation, vol. 204, no. 1, pp. 317-324, 2008.

[17] C. Bai, "Global stability of almost periodic solutions of Hopfield neural networks with neutral time-varying delays," Applied Mathematics and Computation, vol. 203, no. 1, pp. 72-79, 2008.

[18] B. Xiao, "Existence and uniqueness of almost periodic solutions for a class of Hopfield neural networks with neutral delays," Applied Mathematics Letters, vol. 22, no. 4, pp. 528-533, 2009.

[19] H. Xiang and J. Cao, "Almost periodic solution of CohenGrossberg neural networks with bounded and unbounded delays," Nonlinear Analysis: Real World Applications, vol. 10, no. 4, pp. 2407-2419, 2009.

[20] K. Wang and Y. Zhu, "Stability of almost periodic solution for a generalized neutral-type neural networks with delays," Neurocomputing, vol. 73, no. 16-18, pp. 3300-3307, 2010.

[21] J. Liu and G. Zong, "New delay-dependent asymptotic stability conditions concerning BAM neural networks of neutral type," Neurocomputing, vol. 72, no. 10-12, pp. 2549-2555, 2009.

[22] R. Samli and S. Arik, "New results for global stability of a class of neutral-type neural systems with time delays," Applied Mathematics and Computation, vol. 210, no. 2, pp. 564-570, 2009.

[23] R. Samidurai, S. M. Anthoni, and K. Balachandran, "Global exponential stability of neutral-type impulsive neural networks with discrete and distributed delays," Nonlinear Analysis: Hybrid Systems, vol. 4, no. 1, pp. 103-112, 2010.

[24] R. Rakkiyappan, P. Balasubramaniam, and J. Cao, "Global exponential stability results for neutral-type impulsive neural networks," Nonlinear Analysis: Real World Applications, vol. 11, no. 1, pp. 122-130, 2010.

[25] Y. Li, L. Zhao, and X. Chen, "Existence of periodic solutions for neutral type cellular neural networks with delays," Applied Mathematical Modelling, vol. 36, no. 3, pp. 1173-1183, 2012.

[26] P. Balasubramaniam and V. Vembarasan, "Asymptotic stability of BAM neural networks of neutral-type with impulsive effects and time delay in the leakage term," International Journal of Computer Mathematics, vol. 88, no. 15, pp. 3271-3291, 2011.

[27] B. Aulbach and S. Hilger, "Linear dynamic processes with inhomogeneous time scale," in Nonlinear Dynamics and Quantum Dynamical Systems, vol. 59 of Mathematical Research, pp. 9-20, Akademie, Berlin, Germany, 1990.

[28] L. Erbe and S. Hilger, "Sturmian theory on measure chains," Differential Equations and Dynamical Systems, vol. 1, no. 3, pp. 223-244, 1993.

[29] V. Lakshmikantham, S. Sivasundaram, and B. Kaymakcalan, Dynamic Systems on Measure Chains, Kluwer Academic, Dordrecht, The Netherlands, 1996.

[30] R. P. Agarwal and M. Bohner, "Basic calculus on time scales and some of its applications," Results in Mathematics, vol. 35, no. 1-2, pp. 3-22, 1999.

[31] S. Hilger, "Analysis on measure chains-a unified approach to continuous and discrete calculus," Results in Mathematics, vol. 18, no. 1-2, pp. 18-56, 1990.

[32] M. Bohner and A. Peterson, Dynamic Equations on Time Scales: An Introduction with Applications, Birkhäuser, Boston, Mass, USA, 2001.
[33] Y. Li and C. Wang, "Almost periodic functions on time scales and applications," Discrete Dynamics in Nature and Society, vol. 2011, Article ID 727068, 20 pages, 2011.

[34] Y. Li and C. Wang, "Uniformly almost periodic functions and almost periodic solutions to dynamic equations on time scales," Abstract and Applied Analysis, vol. 2011, Article ID 341520, 22 pages, 2011.

[35] J. Zhang, M. Fan, and H. Zhu, "Existence and roughness of exponential dichotomies of linear dynamic equations on time scales," Computers \& Mathematics with Applications, vol. 59, no. 8, pp. 2658-2675, 2010.

[36] V. Lakshmikantham and A. S. Vatsala, "Hybrid systems on time scales," Journal of Computational and Applied Mathematics, vol. 141, no. 1-2, pp. 227-235, 2002.

[37] Y. Li and K. Zhao, "Robust stability of delayed reaction-diffusion recurrent neural networks with Dirichlet boundary conditions on time scales," Neurocomputing, vol. 74, no. 10, pp. 1632-1637, 2011.

[38] Y. K. Li, K. H. Zhao, and Y. Ye, "Stability of reaction-diffusion recurrent neural networks with distributed delays and neumann boundary conditions on time scales," Neural Processing Letters, vol. 36, pp. 217-234, 2012.

[39] J. Shen and J. Cao, "Consensus of multi-agent systems on time scales," IMA Journal of Mathematical Control and Information, vol. 29, no. 4, pp. 507-517, 2012.

[40] Y. K. Li, "Periodic solutions of non-autonomous cellular neural networks with impulses and delays on time scales," IMA Journal of Mathematical Control and Information, 2013. 


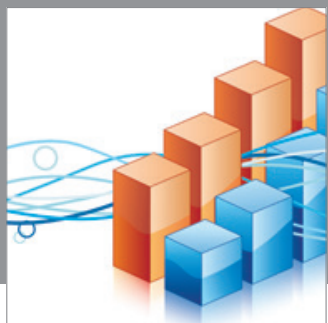

Advances in

Operations Research

mansans

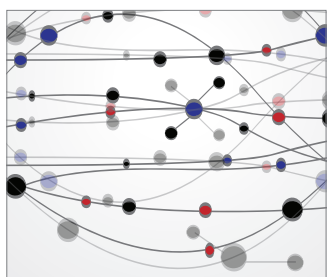

The Scientific World Journal
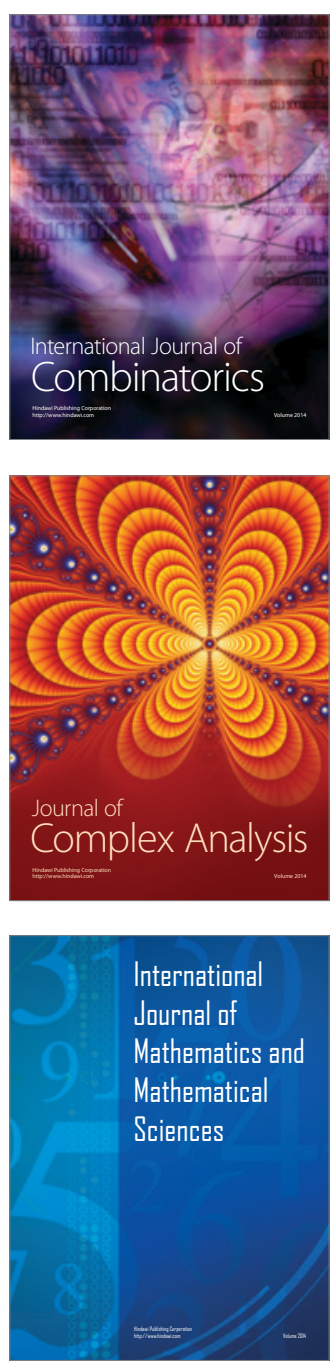
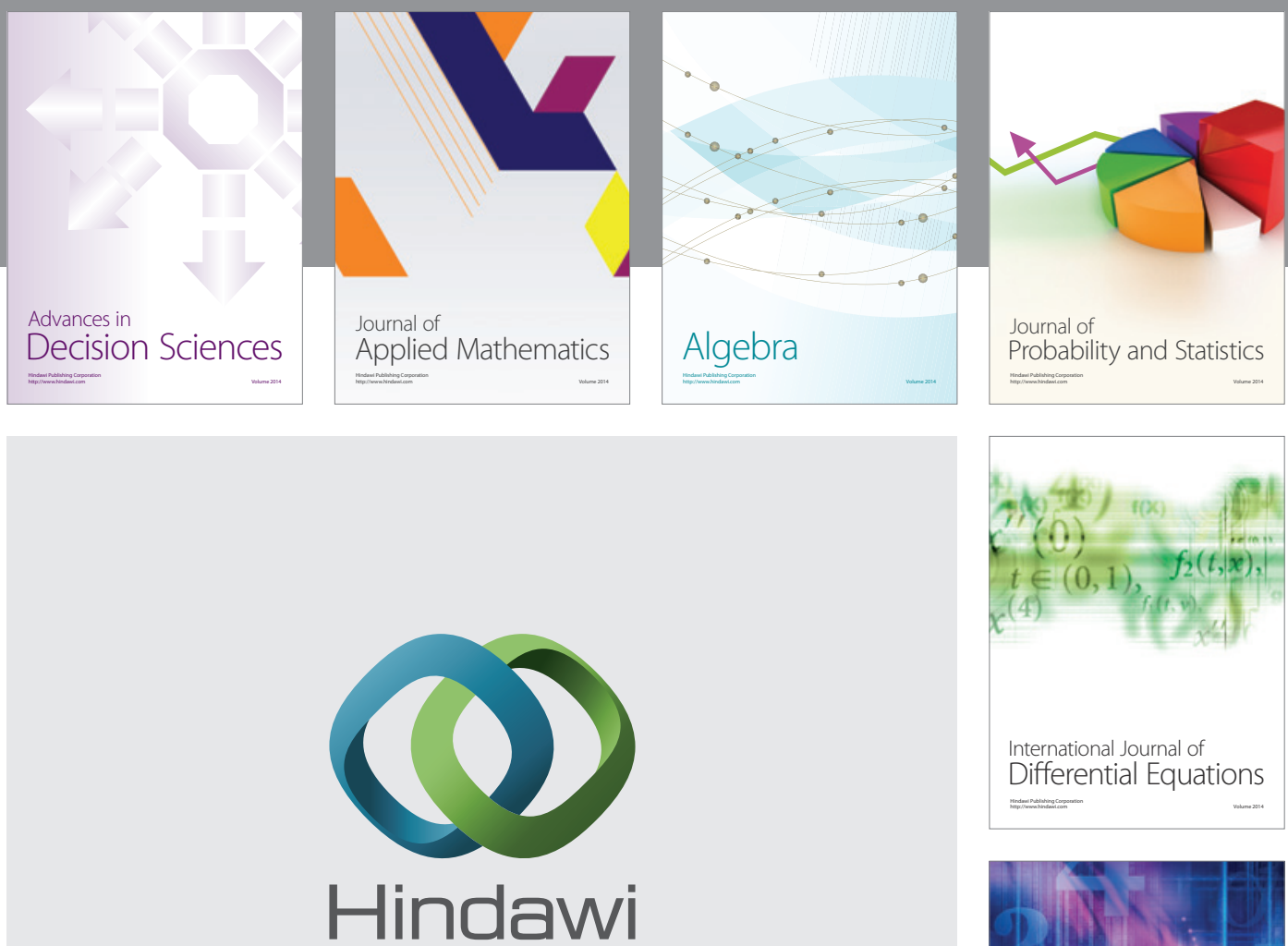

Submit your manuscripts at http://www.hindawi.com
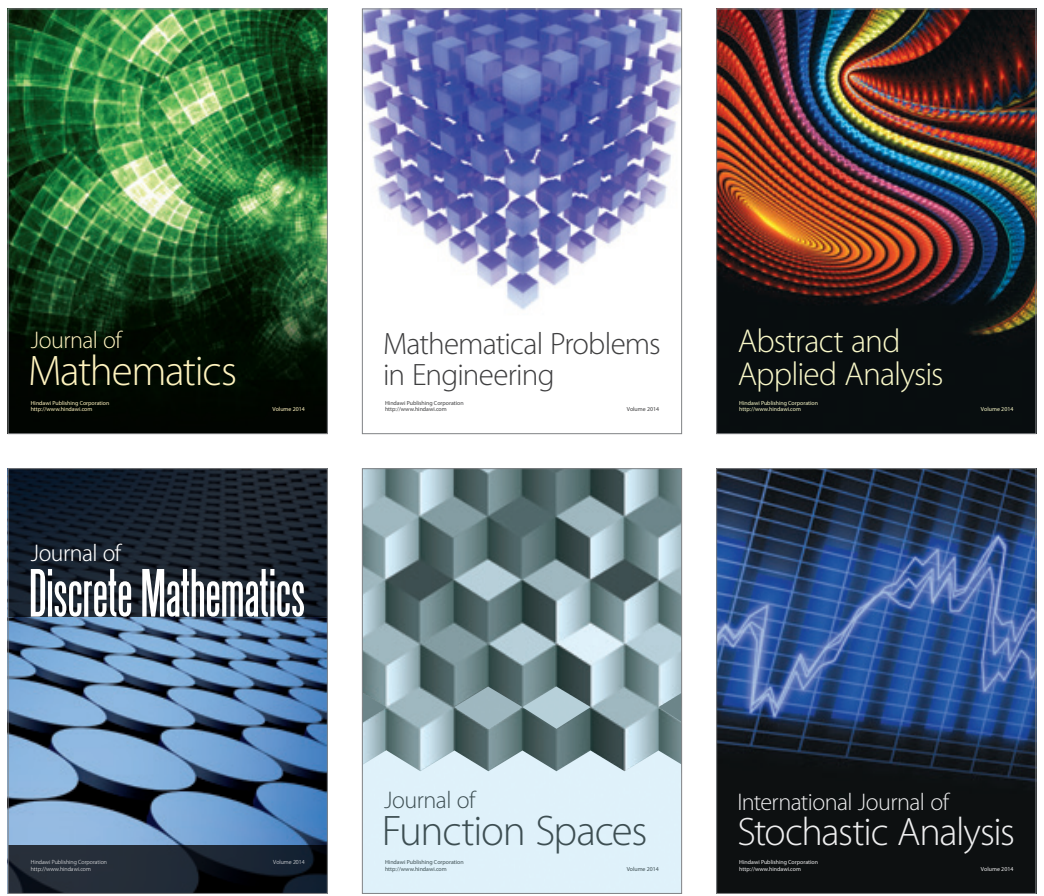

Journal of

Function Spaces

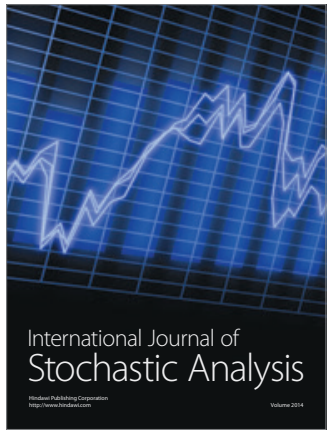

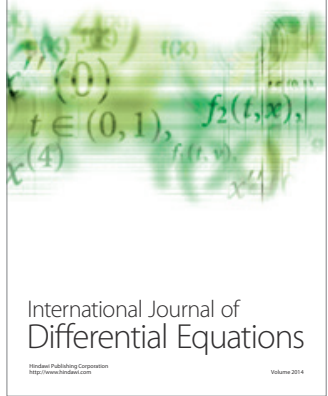
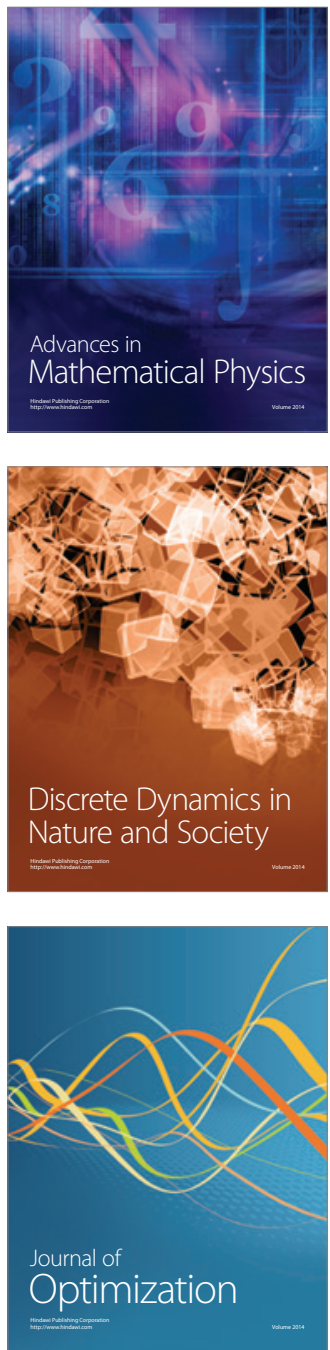